Theoretical and Computational Fluid Dynamics

Volume 21, Number 4 / juillet 2007: 245-269

http://dx.doi.org/10.1007/s00162-007-0047-0

(C2007 Springer Science+Business Media
Archimer, archive institutionnelle de l'Ifremer http://www. ffremer.fr/docelec/

The original publication is available at http://www.springerlink.com

\title{
Comparison between three-dimensional linear and nonlinear tsunami generation models
}

\author{
Youen Kervella ${ }^{1}$, Denys Dutykh ${ }^{2}$ and Frédéric Dias ${ }^{2}$
}

(1) IFREMER, Laboratoire DYNECO/PHYSED, BP 70, 29280 Plouzané, France

(2) CMLA, ENS Cachan, CNRS, PRES UniverSud, 61 Av. President Wilson, 94230 Cachan, France

*: Corresponding author : dias@cmla.ens-cachan.fr

\begin{abstract}
:
The modeling of tsunami generation is an essential phase in understanding tsunamis. For tsunamis generated by underwater earthquakes, it involves the modeling of the sea bottom motion as well as the resulting motion of the water above. A comparison between various models for three-dimensional water motion, ranging from linear theory to fully nonlinear theory, is performed. It is found that for most events the linear theory is sufficient. However, in some cases, more-sophisticated theories are needed. Moreover, it is shown that the passive approach in which the seafloor deformation is simply translated to the ocean surface is not always equivalent to the active approach in which the bottom motion is taken into account, even if the deformation is supposed to be instantaneous.
\end{abstract}

Keywords: Tsunami generation - Finite-volume method - Boundary element method - Water waves Potential flow - Nonlinear shallow water equations 


\title{
Comparison between three-dimensional linear and nonlinear tsunami generation models
}

\author{
Youen Kervella * Denys Dutykh $^{\dagger} \quad$ Frédéric Dias $^{\dagger}$
}

1 February 2007

\begin{abstract}
The modeling of tsunami generation is an essential phase in understanding tsunamis. For tsunamis generated by underwater earthquakes, it involves the modeling of the sea bottom motion as well as the resulting motion of the water above it. A comparison between various models for three-dimensional water motion, ranging from linear theory to fully nonlinear theory, is performed. It is found that for most events the linear theory is sufficient. However, in some cases, more sophisticated theories are needed. Moreover, it is shown that the passive approach in which the seafloor deformation is simply translated to the ocean surface is not always equivalent to the active approach in which the bottom motion is taken into account, even if the deformation is supposed to be instantaneous.
\end{abstract}

\section{Introduction}

Tsunami wave modeling is a challenging task. In particular, it is essential to understand the first minutes of a tsunami, its propagation and finally the resulting inundation and impact on structures. The focus of the present paper is on the generation process. There are different natural phenomena that can lead to a tsunami. For example, one can mention submarine mass failures, slides, volcanic eruptions, falls of asteroids, etc. We refer to the recent review on tsunami science [28] for a complete bibliography on the topic. The present work focuses on tsunami generation by earthquakes.

Two steps in modeling are necessary for an accurate description of tsunami generation: a model for the earthquake fed by the various seismic parameters, and a model for the formation of surface gravity waves resulting from the deformation of the seafloor. In the absence of sophisticated source models, one often uses analytical solutions based on dislocation theory in an elastic half-space for

\footnotetext{
*IFREMER, Laboratoire DYNECO/PHYSED, BP 70, 29280 Plouzané, France

${ }^{\dagger}$ Centre de Mathématiques et de Leurs Applications, École Normale Supérieure de Cachan, 61 avenue du Président Wilson, 94235 Cachan cedex, France; e-mail: dias@cmla.ens-cachan.fr, phone: +331474059 00, fax: +33147405901
} 
the seafloor displacement [26]. For the resulting water motion, the standard practice is to transfer the inferred seafloor displacement to the free surface of the ocean. In this paper, we will call this approach the passive generation approach. ${ }^{1}$ This approach leads to a well-posed initial value problem with zero velocity. An open question for tsunami forecasting modelers is the validity of neglecting the initial velocity. In a recent note, Dutykh et al. [5] used linear theory to show that indeed differences may exist between the standard passive generation and the active generation that takes into account the dynamics of seafloor displacement. The transient wave generation due to the coupling between the seafloor motion and the free surface has been considered by a few authors only. One of the reasons is that it is commonly assumed that the source details are not important. ${ }^{2}$ Ben-Menahem and Rosenman [1] calculated the two-dimensional radiation pattern from a moving source (linear theory). Tuck and Hwang [33] solved the linear long-wave equation in the presence of a moving bottom and a uniformly sloping beach. Hammack [15] generated waves experimentally by raising or lowering a box at one end of a channel. According to Synolakis and Bernard [28], Houston and Garcia [17] were the first to use more geophysically realistic initial conditions. For obvious reasons, the quantitative differences in the distribution of seafloor displacement due to underwater earthquakes compared with more conventional earthquakes are still poorly known. Villeneuve and Savage [35] derived model equations which combine the linear effect of frequency dispersion and the nonlinear effect of amplitude dispersion, and included the effects of a moving bed. Todorovska and Trifunac [31] considered the generation of tsunamis by a slowly spreading uplift of the seafloor.

In this paper, we mostly follow the standard passive generation approach. Several tsunami generation models and numerical methods suited for these models are presented and compared. The focus of our work is on modelling the fluid motion. It is assumed that the seabed deformation satisfies all the necessary hypotheses required to apply Okada's solution. The main objective is to confirm or infirm the lack of importance of nonlinear effects and/or frequency dispersion in tsunami generation. This result may have implications in terms of computational cost. The goal is to optimize the ratio between the complexity of the model and the accuracy of the results. Government agencies need to compute accurately tsunami propagation in real time in order to know where to evacuate people. Therefore any saving in computational time is crucial (see for example the code MOST used by the National Oceanic and Atmospheric Administration in the US [29] or the code TUNAMI developed by the Disaster Control Research Center in Japan). Liu and Liggett [24] already performed comparisons between linear and nonlinear water waves but their study was restricted

\footnotetext{
${ }^{1}$ In the pioneering paper [18], Kajiura analyzed the applicability of the passive approach using Green's functions. In the tsunami literature, this approach is sometimes called the piston model of tsunami generation.

${ }^{2}$ As pointed out by Geist et al. [8], the 2004 Indian Ocean tsunami shed some doubts about this belief. The measurements from land based stations that use the Global Positioning System to track ground movements revealed that the fault continued to slip after it stopped releasing seismic energy. Even though this slip was relatively slow, it contributed to the tsunami and may explain the surprising tsunami heights.
} 
to simple bottom deformations, namely the generation of transient waves by an upthrust of a rectangular block, and the nonlinear computations were restricted to two-dimensional flows. Bona et al. [3] assessed how well a model equation with weak nonlinearity and dispersion describes the propagation of surface water waves generated at one end of a long channel. In their experiments, they found that the inclusion of a dissipative term was more important than the inclusion of nonlinearity, although the inclusion of nonlinearity was undoubtedly beneficial in describing the observations. The importance of dispersive effects in tsunami propagation is not directly addressed in the present paper. Indeed these effects cannot be measured without taking into account the duration (or distance) of tsunami propagation [32].

The paper is organized as follows. In Section 2, we review the equations that are commonly used for water-wave propagation, namely the fully nonlinear potential flow (FNPF) equations. Section 3 provides a description of the linear theory, with explicit expressions for the free-surface elevation and the velocities everywhere inside the fluid domain, both for active and passive generations. Section 4 is devoted to the nonlinear shallow water (NSW) equations and their numerical integration by a finite volume scheme. In Section 5 we briefly describe the boundary element numerical method used to integrate the FNPF equations. The following section (Section 6) is devoted to comparisons between the various models and a discussion on the results. The main conclusion is that linear theory is sufficient in general but that passive generation overestimates the initial transient waves in some cases. Finally directions for future research are outlined.

\section{Physical problem description}

In the whole paper, the vertical coordinate is denoted by $z$, while the two horizontal coordinates are denoted by $x$ and $y$, respectively. The sea bottom deformation following an underwater earthquake is a complex phenomenon. This is why, for theoretical or experimental studies, researchers have often used simplified bottom motions such as the vertical motion of a box. In order to determine the deformations of the sea bottom due to an earthquake, we use the analytical solution obtained for a dislocation in an elastic half-space [26]. This solution, which at present time is used by the majority of tsunami wave modelers to produce an initial condition for tsunami propagation simulations, provides an explicit expression of the bottom surface deformation that depends on a dozen of source parameters such as the dip angle $\delta$, fault depth $d_{f}$, fault dimensions (length and width), Burger's vector D, Young's modulus, Poisson ratio, etc. Some of these parameters are shown in figure 1. More details can be found in [4] for example. A value of $90^{\circ}$ for the dip angle corresponds to a vertical fault. Varying the fault slip $|\mathbf{D}|$ does not change the co-seismic deformation pattern, only its magnitude. The values of the parameters used in the present paper are given in Table 1. A typical dip-slip solution is shown in figure 2 (the angle $\phi$ is equal to 0 , while the rake angle $\theta$ is equal to $\pi / 2$ ). 


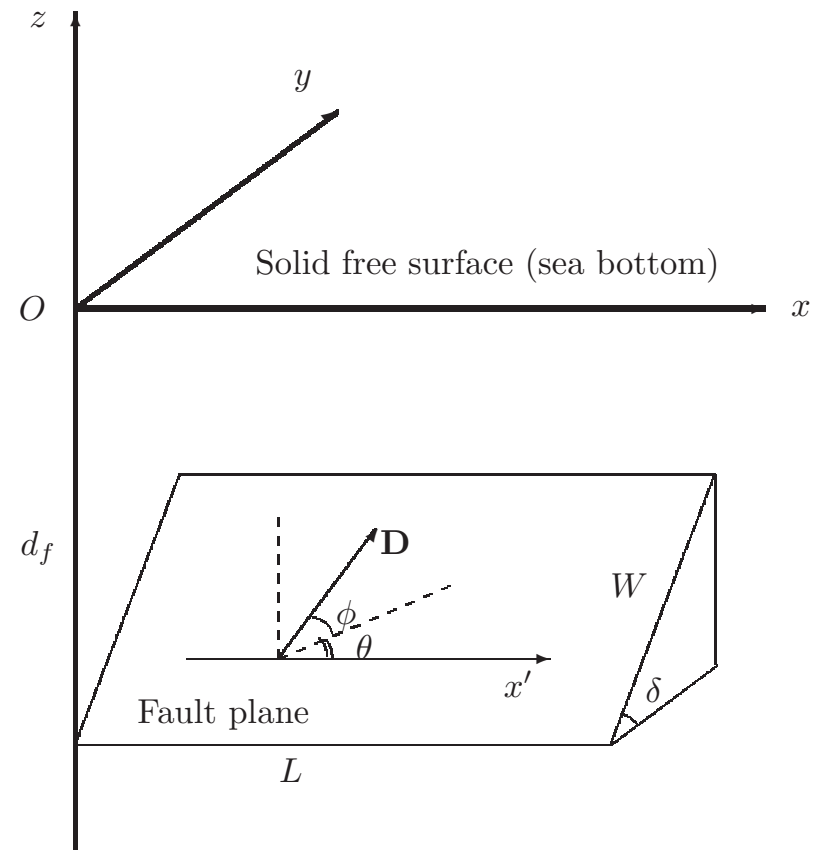

Figure 1: Geometry of the source model (dip angle $\delta$, depth $d_{f}$, length $L$, width $W$ ) and orientation of Burger's vector $\mathbf{D}$ (rake angle $\theta$, angle $\phi$ between the fault plane and Burger's vector).

\begin{tabular}{lc} 
parameter & value \\
\hline Dip angle $\delta$ & $13^{\circ}$ \\
Fault depth $d_{f}, \mathrm{~km}$ & 3 \\
Fault length $L, \mathrm{~km}$ & 6 \\
Fault width $W, \mathrm{~km}$ & 4 \\
Magnitude of Burger's vector $|\mathbf{D}|, \mathrm{m}$ & 1 \\
Young's modulus $E$, GPa & 9.5 \\
Poisson ratio $\nu$ & 0.23 \\
\hline
\end{tabular}

Table 1: Typical parameter set for the source used to model the seafloor deformation due to an earthquake in the present study. The dip angle, Young's modulus and Poisson ratio correspond roughly to those of the 2004 Sumatra event. The fault depth, length and width, as well as the magnitude of Burger's vector, have been reduced for computation purposes. 


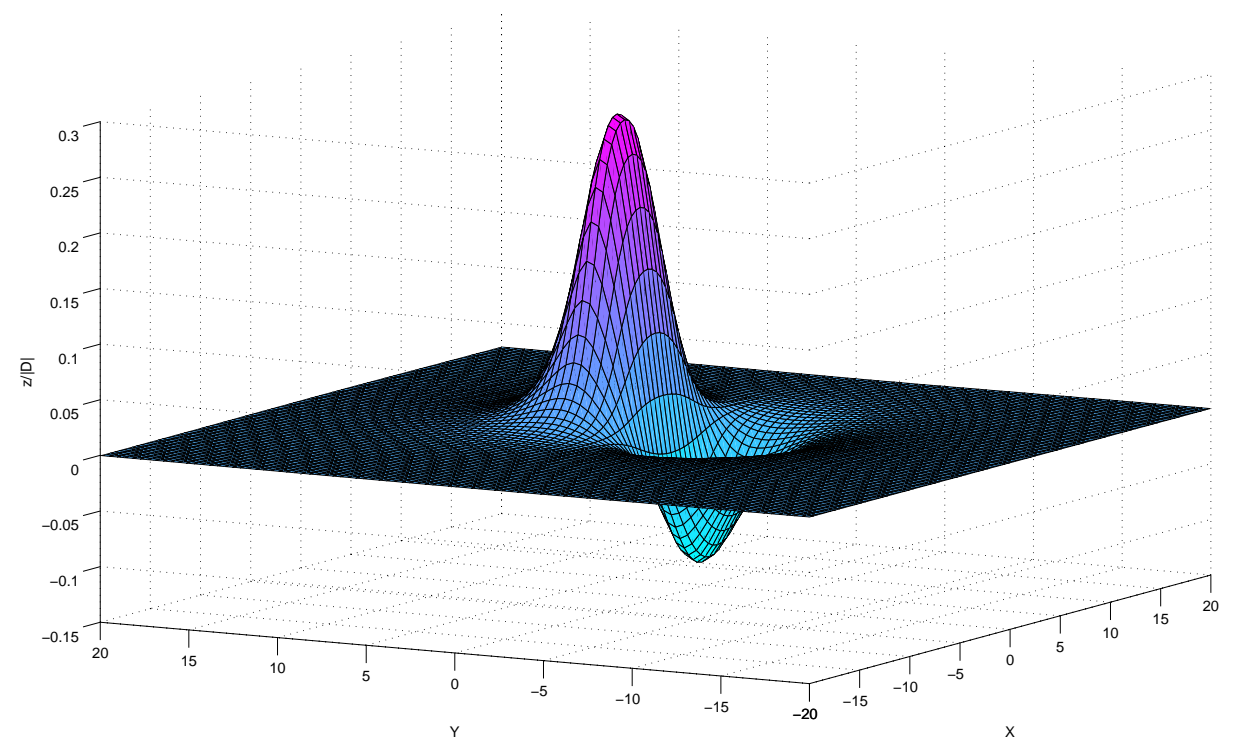

Figure 2: Typical seafloor deformation due to dip-slip faulting. The parameters are those of Table 1 . The distances along the horizontal axes $x$ and $y$ are expressed in kilometers. 


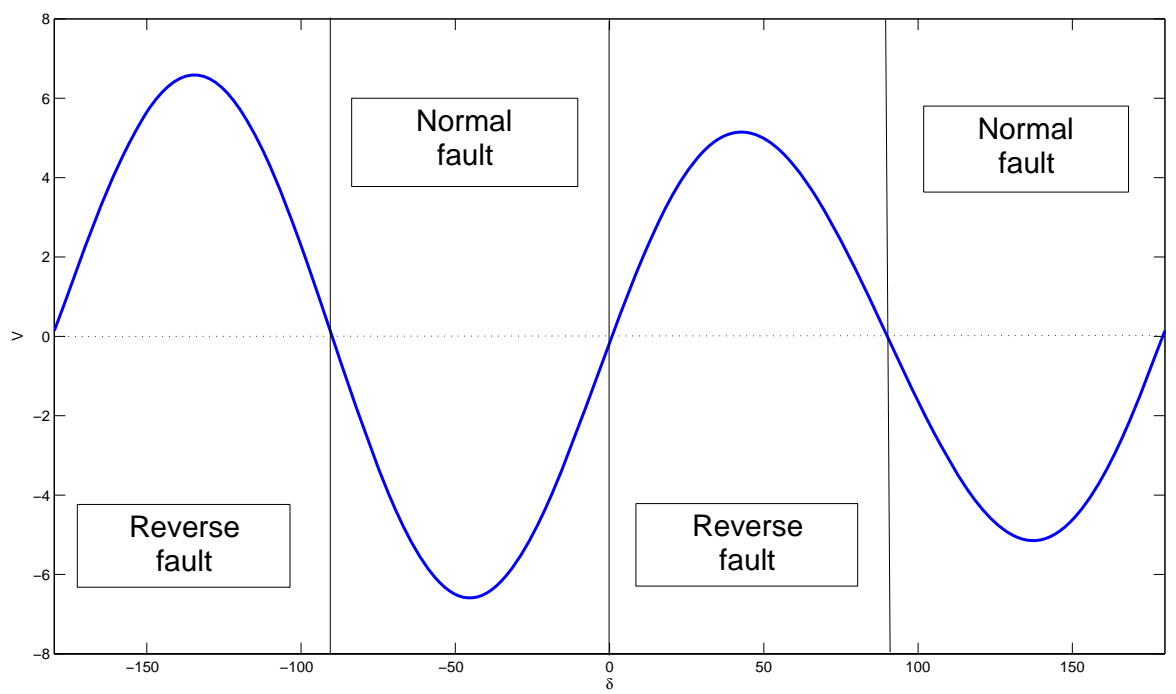

Figure 3: Initial net volume $V\left(\right.$ in $\left.\mathrm{km}^{3}\right)$ of the seafloor displacement as a function of the dip angle $\delta\left(\right.$ in $\left.^{\circ}\right)$. All the other parameters, which are given in Table 1, are kept constant.

Let $z=\zeta(x, y, t)$ denote the deformation of the sea bottom. Hammack and Segur [16] suggested that there are two main kinds of behaviour for the generated waves depending on whether the net volume $V$ of the initial bottom surface deformation

$$
V=\int_{\mathbb{R}^{2}} \zeta(x, y, 0) d x d y
$$

is positive or not. ${ }^{3}$ A positive $V$ is achieved for example for a "reverse fault", i.e. when the dip angle $\delta$ satisfies $0 \leq \delta \leq \pi / 2$ or $-\pi \leq \delta \leq-\pi / 2$, as shown in figure 3. A negative $V$ is achieved for a "normal fault", i.e. when the dip angle $\delta$ satisfies $\pi / 2 \leq \delta \leq \pi$ or $-\pi / 2 \leq \delta \leq 0$.

The conclusions of [16] are based on the Korteweg-de Vries (KdV) equation and were in part confirmed by their experiments. If $V$ is positive, waves of stable form (solitons) evolve and are followed by a dispersive train of oscillatory waves, regardless of the exact structure of $\zeta(x, y, 0)$. If $V$ is negative, and if the initial data is non-positive everywhere, no solitons evolve. But, if $V$ is negative and there is a region of elevation in the initial data (which corresponds to a typical Okada solution for a normal fault), solitons can evolve and we have checked

\footnotetext{
${ }^{3}$ However it should be noted that the analysis of [16] is restricted to one-dimensional unidirectional waves. We assume here that their conclusions can be extended to two-dimensional bi-directional waves.
} 


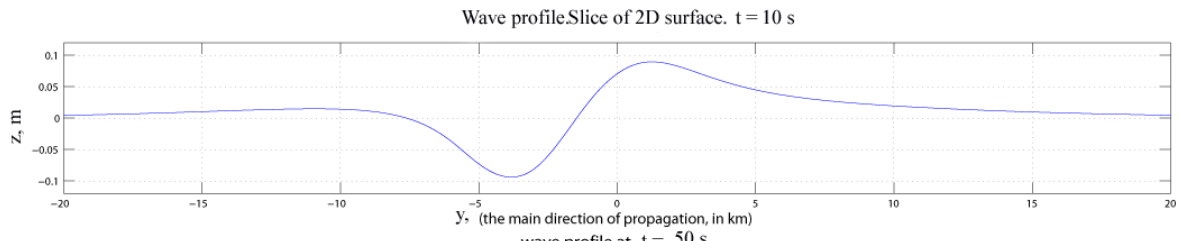
wave profile at $\mathrm{t}=50 \mathrm{~s}$

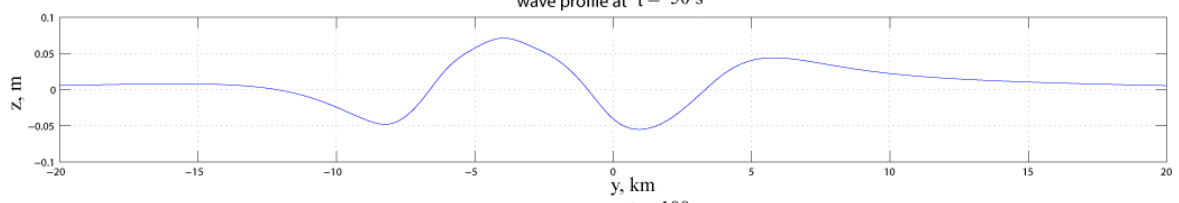

wave profile at $\mathrm{t}=100 \mathrm{~s}$

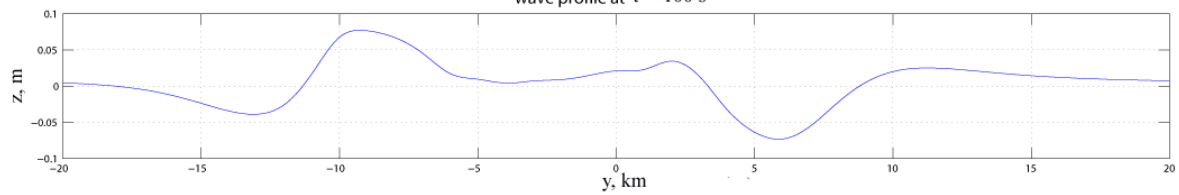

wave profile at $t=150 \mathrm{~s}$

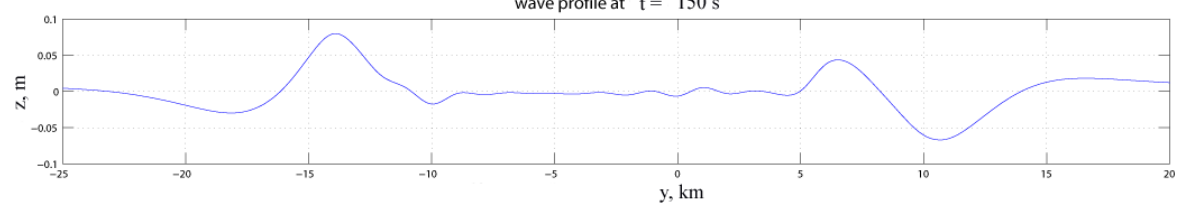

Figure 4: Wave profiles at different times for the case of a normal fault ( $\delta=$ $167^{\circ}$ ). The seafloor deformation occurs instantaneously at $t=0$. The water depth $h(x, y)$ is assumed to be constant.

this last result using the FNPF equations (see figure 4). In this study, we focus on the case where $V$ is positive with a dip angle $\delta$ equal to $13^{\circ}$, according to the seismic data of the 26 December 2004 Sumatra-Andaman event (see for example [22]). However, the sea bottom deformation often has an $N$-shape, with subsidence on one side of the fault and uplift on the other side as shown in figure 2. In that case, one may expect the positive $V$ behaviour on one side and the negative $V$ behaviour on the other side. Recall that the experiments of Hammack and Segur [16] were performed in the presence of a vertical wall next to the moving bottom and their analysis was based on the uni-directional $\mathrm{KdV}$ wave equation.

We now consider the fluid domain. A sketch is shown in figure 5. The fluid domain $\Omega$ is bounded above by the free surface and below by the rigid ocean floor. It is unbounded in the horizontal $x-$ and $y$ - directions. So, one can write

$$
\Omega=\mathbb{R}^{2} \times[-h(x, y)+\zeta(x, y, t), \eta(x, y, t)] .
$$

Before the earthquake the fluid is assumed to be at rest, thus the free surface and the solid boundary are defined by $z=0$ and $z=-h(x, y)$, respectively. For 


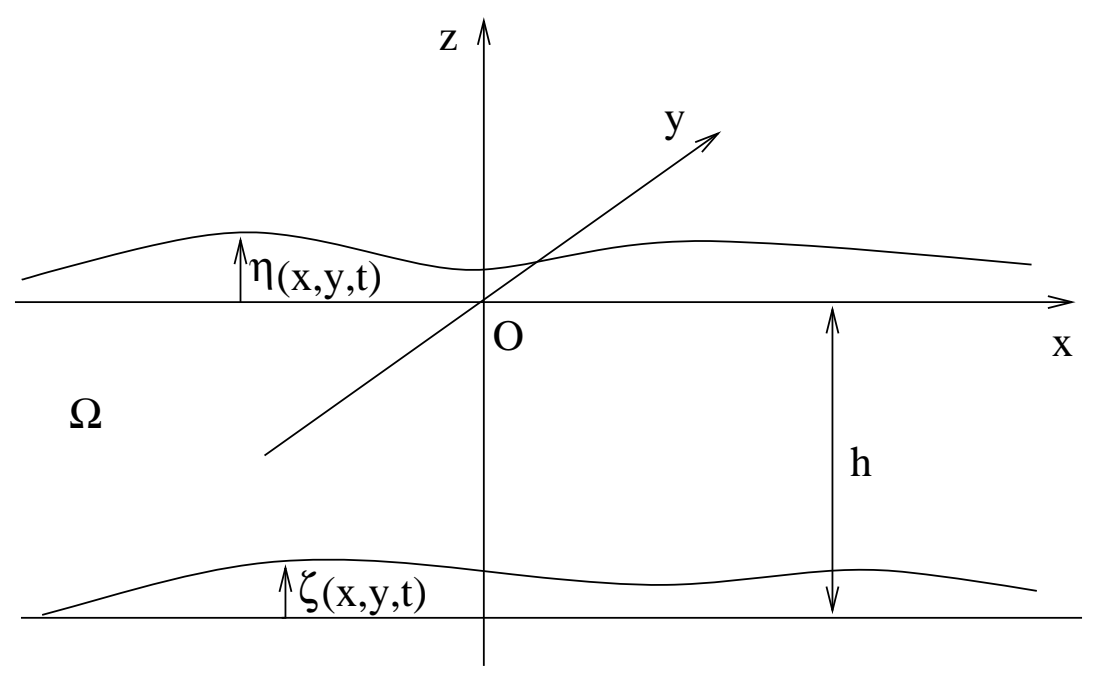

Figure 5: Definition of the fluid domain $\Omega$ and of the coordinate system $(x, y, z)$.

simplicity $h(x, y)$ is assumed to be a constant. Of course, in real situations, this is never the case but for our purpose the bottom bathymetry is not important. Starting at time $t=0$, the solid boundary moves in a prescribed manner which is given by

$$
z=-h+\zeta(x, y, t), \quad t \geq 0 .
$$

The deformation of the sea bottom is assumed to have all the necessary properties needed to compute its Fourier transform in $x, y$ and its Laplace transform in $t$. The resulting deformation of the free surface $z=\eta(x, y, t)$ is to be found as part of the solution. It is also assumed that the fluid is incompressible and the flow irrotational. The latter implies the existence of a velocity potential $\phi(x, y, z, t)$ which completely describes the flow. By definition of $\phi$ the fluid velocity vector can be expressed as $\mathbf{q}=\nabla \phi$. Thus, the continuity equation becomes

$$
\nabla \cdot \mathbf{q}=\Delta \phi=0, \quad(x, y, z) \in \Omega .
$$

The potential $\phi(x, y, z, t)$ must satisfy the following kinematic boundary conditions on the free surface and the solid boundary, respectively:

$$
\begin{aligned}
& \frac{\partial \phi}{\partial z}=\frac{\partial \eta}{\partial t}+\frac{\partial \phi}{\partial x} \frac{\partial \eta}{\partial x}+\frac{\partial \phi}{\partial y} \frac{\partial \eta}{\partial y}, \quad z=\eta(x, y, t) \\
& \frac{\partial \phi}{\partial z}=\frac{\partial \zeta}{\partial t}+\frac{\partial \phi}{\partial x} \frac{\partial \zeta}{\partial x}+\frac{\partial \phi}{\partial y} \frac{\partial \zeta}{\partial y}, \quad z=-h+\zeta(x, y, t) .
\end{aligned}
$$

Further assuming the flow to be inviscid and neglecting surface tension effects, one can write the dynamic condition to be satisfied on the free surface as

$$
\frac{\partial \phi}{\partial t}+\frac{1}{2}|\nabla \phi|^{2}+g \eta=0, \quad z=\eta(x, y, t)
$$


where $g$ is the acceleration due to gravity. The atmospheric pressure has been chosen as reference pressure.

The equations are more transparent when written in dimensionless variables. However the choice of the reference lengths and speeds is subtle. Different choices lead to different models. Let the new independent variables be

$$
\tilde{x}=x / \lambda, \quad \tilde{y}=y / \lambda, \quad \tilde{z}=z / d, \quad \tilde{t}=c_{0} t / \lambda,
$$

where $\lambda$ is the horizontal scale of the motion and $d$ a typical water depth. The speed $c_{0}$ is the long wave speed based on the depth $d\left(c_{0}=\sqrt{g d}\right)$. Let the new dependent variables be

$$
\tilde{\eta}=\frac{\eta}{a}, \quad \tilde{\zeta}=\frac{\zeta}{a}, \quad \tilde{\phi}=\frac{c_{0}}{a g \lambda} \phi,
$$

where $a$ is a characteristic wave amplitude.

In dimensionless form, and after dropping the tildes, the equations become

$$
\begin{gathered}
\frac{\partial^{2} \phi}{\partial z^{2}}+\mu^{2}\left(\frac{\partial^{2} \phi}{\partial x^{2}}+\frac{\partial^{2} \phi}{\partial y^{2}}\right)=0, \quad(x, y, z) \in \Omega \\
\frac{\partial \phi}{\partial z}=\mu^{2} \frac{\partial \eta}{\partial t}+\varepsilon \mu^{2}\left(\frac{\partial \phi}{\partial x} \frac{\partial \eta}{\partial x}+\frac{\partial \phi}{\partial y} \frac{\partial \eta}{\partial y}\right), \quad z=\varepsilon \eta(x, y, t) \\
\frac{\partial \phi}{\partial z}=\mu^{2} \frac{\partial \zeta}{\partial t}+\varepsilon \mu^{2}\left(\frac{\partial \phi}{\partial x} \frac{\partial \zeta}{\partial x}+\frac{\partial \phi}{\partial y} \frac{\partial \zeta}{\partial y}\right), \quad z=-\frac{h}{d}+\varepsilon \zeta(x, y, t) \\
\mu^{2} \frac{\partial \phi}{\partial t}+\frac{1}{2} \varepsilon\left(\mu^{2}\left(\frac{\partial \phi}{\partial x}\right)^{2}+\mu^{2}\left(\frac{\partial \phi}{\partial y}\right)^{2}+\left(\frac{\partial \phi}{\partial z}\right)^{2}\right)+\mu^{2} \eta=0, \quad z=\varepsilon \eta(x, y, t)
\end{gathered}
$$

where two dimensionless numbers have been introduced:

$$
\varepsilon=a / d, \quad \mu=d / \lambda .
$$

For the propagation of tsunamis, both numbers $\varepsilon$ and $\mu$ are small. Indeed the satellite altimetry observations of the 2004 Boxing Day tsunami waves obtained by two satellites that passed over the Indian Ocean a couple of hours after the rupture process occurred gave an amplitude $a$ of roughly $60 \mathrm{~cm}$ in the open ocean. The typical wavelength estimated from the width of the segments that experienced slip is between 160 and $240 \mathrm{~km}$ [22]. The water depth ranges from 4 $\mathrm{km}$ towards the west of the rupture to $1 \mathrm{~km}$ towards the east. Therefore average values for $\varepsilon$ and $\mu$ in the open ocean are $\varepsilon \approx 2 \times 10^{-4}$ and $\mu \approx 2 \times 10^{-2}$. A more precise range for these two dimensionless numbers is

$$
1.5 \times 10^{-4}<\varepsilon<6 \times 10^{-4}, \quad 4 \times 10^{-3}<\mu<2.5 \times 10^{-2} .
$$

The water-wave problem, either in the form of an initial value problem (IVP) or in the form of a boundary value problem (BVP), is difficult to solve because of the nonlinearities in the boundary conditions and the unknown computational domain. 


\section{$3 \quad$ Linear theory}

First we perform the linearization of the above equations and boundary conditions. It is equivalent to taking the limit of (3)-(6) as $\varepsilon \rightarrow 0$. The linearized problem can also be obtained by expanding the unknown functions as power series of the small parameter $\varepsilon$. Collecting terms of the lowest order in $\varepsilon$ yields the linear approximation. For the sake of convenience, we now switch back to the physical variables. The linearized problem in dimensional variables reads

$$
\begin{gathered}
\Delta \phi=0, \quad(x, y, z) \in \mathbb{R}^{2} \times[-h, 0], \\
\frac{\partial \phi}{\partial z}=\frac{\partial \eta}{\partial t}, \quad z=0, \\
\frac{\partial \phi}{\partial z}=\frac{\partial \zeta}{\partial t}, \quad z=-h, \\
\frac{\partial \phi}{\partial t}+g \eta=0, \quad z=0 .
\end{gathered}
$$

The bottom motion appears in equation (11). Combining equations (10) and (12) yields the single free-surface condition

$$
\frac{\partial^{2} \phi}{\partial t^{2}}+g \frac{\partial \phi}{\partial z}=0, \quad z=0 .
$$

Most studies of tsunami generation assume that the initial free-surface deformation is equal to the vertical displacement of the ocean bottom and take a zero velocity field as initial condition. The details of wave motion are completely neglected during the time that the source operates. While tsunami modelers often justify this assumption by the fact that the earthquake rupture occurs very rapidly, there are some specific cases where the time scale and/or the horizontal extent of the bottom deformation may become an important factor. This was emphasized for example by Todorovska and Trifunac [31] and Todorovska et al. [30], who considered the generation of tsunamis by a slowly spreading uplift of the seafloor in order to explain some observations related to past tsunamis. However they did not use realistic source models.

Our claim is that it is important to make a distinction between two mechanisms of generation: an active mechanism in which the bottom moves according to a given time law and a passive mechanism in which the seafloor deformation is simply translated to the free surface. Recently Dutykh et al. [5] showed that even in the case of an instantaneous seafloor deformation, there may be differences between these two generation processes.

\subsection{Active generation}

Since in this case the system is assumed to be at rest at $t=0$, the initial condition simply is

$$
\eta(x, y, 0) \equiv 0 .
$$


In fact, $\eta(x, y, t)=0$ for all times $t<0$ and the same condition holds for the velocities. For $t<0$, the water is at rest and the bottom motion is such that $\zeta(x, y, t)=0$ for $t<0$.

The problem (9)-(13) can be solved by using the method of integral transforms. We apply the Fourier transform in $(x, y)$,

$$
\mathfrak{F}[f]=\widehat{f}(k, \ell)=\int_{\mathbb{R}^{2}} f(x, y) e^{-i(k x+\ell y)} d x d y,
$$

with its inverse transform

$$
\mathfrak{F}^{-1}[\widehat{f}]=f(x, y)=\frac{1}{(2 \pi)^{2}} \int_{\mathbb{R}^{2}} \widehat{f}(k, \ell) e^{i(k x+\ell y)} d k d \ell,
$$

and the Laplace transform in time $t$,

$$
\mathfrak{L}[g]=\mathbf{g}(s)=\int_{0}^{+\infty} g(t) e^{-s t} d t .
$$

For the combined Fourier and Laplace transforms, the following notation is introduced:

$$
\mathfrak{F} \mathfrak{L}[F(x, y, t)]=\bar{F}(k, \ell, s)=\int_{\mathbb{R}^{2}} e^{-i(k x+\ell y)} d x d y \int_{0}^{+\infty} F(x, y, t) e^{-s t} d t .
$$

After applying the transforms, equations (9), (11) and (13) become

$$
\begin{gathered}
\frac{d^{2} \bar{\phi}}{d z^{2}}-\left(k^{2}+\ell^{2}\right) \bar{\phi}=0, \\
\frac{d \bar{\phi}}{d z}(k, \ell,-h, s)=s \bar{\zeta}(k, \ell, s), \\
s^{2} \bar{\phi}(k, \ell, 0, s)+g \frac{d \bar{\phi}}{d z}(k, \ell, 0, s)=0 .
\end{gathered}
$$

The transformed free-surface elevation can be obtained from (12):

$$
\bar{\eta}(k, \ell, s)=-\frac{s}{g} \bar{\phi}(k, \ell, 0, s) .
$$

A general solution of equation (15) is given by

$$
\bar{\phi}(k, \ell, z, s)=A(k, \ell, s) \cosh (m z)+B(k, \ell, s) \sinh (m z),
$$

where $m=\sqrt{k^{2}+\ell^{2}}$. The functions $A(k, \ell, s)$ and $B(k, \ell, s)$ can be easily found from the boundary conditions (16) and (17):

$$
\begin{aligned}
A(k, \ell, s) & =-\frac{g s \bar{\zeta}(k, \ell, s)}{\cosh (m h)\left[s^{2}+g m \tanh (m h)\right]} \\
B(k, \ell, s) & =\frac{s^{3} \bar{\zeta}(k, \ell, s)}{m \cosh (m h)\left[s^{2}+g m \tanh (m h)\right]} .
\end{aligned}
$$


From now on, the notation

$$
\omega=\sqrt{g m \tanh (m h)}
$$

will be used. Substituting the expressions for the functions $A$ and $B$ in (19) yields

$$
\bar{\phi}(k, \ell, z, s)=-\frac{g s \bar{\zeta}(k, \ell, s)}{\cosh (m h)\left(s^{2}+\omega^{2}\right)}\left(\cosh (m z)-\frac{s^{2}}{g m} \sinh (m z)\right) .
$$

The free-surface elevation (18) becomes

$$
\bar{\eta}(k, \ell, s)=\frac{s^{2} \bar{\zeta}(k, \ell, s)}{\cosh (m h)\left(s^{2}+\omega^{2}\right)} .
$$

Inverting the Laplace and Fourier transforms provides the general integral solution

$$
\eta(x, y, t)=\frac{1}{(2 \pi)^{2}} \iint_{\mathbb{R}^{2}} \frac{e^{i(k x+\ell y)}}{\cosh (m h)} \frac{1}{2 \pi i} \int_{\mu-i \infty}^{\mu+i \infty} \frac{s^{2} \bar{\zeta}(k, \ell, s)}{s^{2}+\omega^{2}} e^{s t} d s d k d \ell .
$$

In some applications it is important to know not only the free-surface elevation but also the velocity field inside the fluid domain. In the present study we consider seabed deformations with the structure

$$
\zeta(x, y, t):=\zeta_{0}(x, y) T(t) .
$$

Mathematically we separate the time dependence from the spatial coordinates. There are two main reasons for doing this. First of all we want to be able to invert analytically the Laplace transform. The second reason is more fundamental. In fact, dynamic source models are not easily available. Okada's solution, which was briefly described in the previous section, provides the static sea-bed deformation $\zeta_{0}(x, y)$. Hammack [15] considered two types of time histories: an exponential and a half-sine bed movements. Dutykh and Dias [4] considered two additional time histories: a linear and an instantaneous bed movements. We show below that taking an instantaneous seabed deformation (in that case the function $T(t)$ is the Heaviside step function) is not equivalent to instantaneously transferring the seabed deformation to the ocean surface ${ }^{4}$.

In equation (21), we obtained the Fourier-Laplace transform of the velocity potential $\phi(x, y, z, t)$ :

$$
\bar{\phi}(k, \ell, z, s)=-\frac{g s \widehat{\zeta_{0}}(k, \ell) \mathbf{T}(s)}{\cosh (m h)\left(s^{2}+\omega^{2}\right)}\left(\cosh (m z)-\frac{s^{2}}{g m} \sinh (m z)\right) .
$$

\footnotetext{
${ }^{4}$ In the framework of the linearized shallow water equations, one can show that it is equivalent to take an instantaneous seabed deformation or to instantaneously transfer the seabed deformation to the ocean surface [33].
} 
Let us evaluate the velocity field at an arbitrary level $z=\beta h$ with $-1 \leq \beta \leq 0$. In the linear approximation the value $\beta=0$ corresponds to the free surface while $\beta=-1$ corresponds to the bottom. Below the horizontal velocities are denoted by $\mathbf{u}$ and the horizontal gradient $(\partial / \partial x, \partial / \partial y)$ is denoted by $\nabla_{h}$. The vertical velocity component is simply $w$. The Fourier transform parameters are denoted by $\mathbf{k}=(k, \ell)$.

Taking the Fourier and Laplace transforms of

$$
\mathbf{u}(x, y, t ; \beta)=\left.\nabla_{h} \phi(x, y, z, t)\right|_{z=\beta h}
$$

yields

$$
\begin{aligned}
\overline{\mathbf{u}}(k, \ell, s ; \beta) & =-i \bar{\phi}(k, \ell, \beta h, s) \mathbf{k} \\
& =i \frac{g s \widehat{\zeta_{0}}(k, \ell) \mathbf{T}(s)}{\cosh (m h)\left(s^{2}+\omega^{2}\right)}\left(\cosh (\beta m h)-\frac{s^{2}}{g m} \sinh (\beta m h)\right) \mathbf{k}
\end{aligned}
$$

Inverting the Fourier and Laplace transforms gives the general formula for the horizontal velocity vector:

$$
\begin{aligned}
\mathbf{u}(x, y, t ; \beta)= & \frac{i g}{4 \pi^{2}} \iint_{\mathbb{R}^{2}} \frac{\mathbf{k} \widehat{\zeta_{0}}(k, \ell) \cosh (m \beta h) e^{i(k x+\ell y)}}{\cosh (m h)} \frac{1}{2 \pi i} \int_{\mu-i \infty}^{\mu+i \infty} \frac{s \mathbf{T}(s) e^{s t}}{s^{2}+\omega^{2}} d s d \mathbf{k} \\
& -\frac{i}{4 \pi^{2}} \iint_{\mathbb{R}^{2}} \frac{\mathbf{k} \widehat{\zeta_{0}}(k, \ell) \sinh (m \beta h) e^{i(k x+\ell y)}}{m \cosh (m h)} \frac{1}{2 \pi i} \int_{\mu-i \infty}^{\mu+i \infty} \frac{s^{3} \mathbf{T}(s) e^{s t}}{s^{2}+\omega^{2}} d s d \mathbf{k} .
\end{aligned}
$$

Next we determine the vertical component of the velocity $w(x, y, t ; \beta)$. It is easy to obtain the Fourier-Laplace transform $\bar{w}(k, \ell, s ; \beta)$ by differentiating (24):

$$
\bar{w}(k, \ell, s ; \beta)=\left.\frac{\partial \bar{\phi}}{\partial z}\right|_{z=\beta h}=\frac{s g \widehat{\zeta_{0}}(k, \ell) \mathbf{T}(s)}{\cosh (m h)\left(s^{2}+\omega^{2}\right)}\left(\frac{s^{2}}{g} \cosh (\beta m h)-m \sinh (\beta m h)\right) .
$$

Inverting this transform yields

$$
\begin{aligned}
w(x, y, t ; \beta)= & \frac{1}{4 \pi^{2}} \iint_{\mathbb{R}^{2}} \frac{\cosh (\beta m h) \widehat{\zeta_{0}}(k, \ell)}{\cosh (m h)} e^{i(k x+\ell y)} \frac{1}{2 \pi i} \int_{\mu-i \infty}^{\mu+i \infty} \frac{s^{3} \mathbf{T}(s) e^{s t}}{s^{2}+\omega^{2}} d s d \mathbf{k} \\
& -\frac{g}{4 \pi^{2}} \iint_{\mathbb{R}^{2}} \frac{m \sinh (\beta m h) \widehat{\zeta_{0}}(k, \ell)}{\cosh (m h)} e^{i(k x+\ell y)} \frac{1}{2 \pi i} \int_{\mu-i \infty}^{\mu+i \infty} \frac{s \mathbf{T}(s) e^{s t}}{s^{2}+\omega^{2}} d s d \mathbf{k},
\end{aligned}
$$

for $-1 \leq \beta \leq 0$.

In the case of an instantaneous seabed deformation, $T(t)=H(t)$, where $H(t)$ denotes the Heaviside step function. The resulting expressions for $\eta, \mathbf{u}$ 
and $w$ (on the free surface), which are valid for $t>0$, are

$$
\begin{aligned}
\eta(x, y, t) & =\frac{1}{(2 \pi)^{2}} \iint_{\mathbb{R}^{2}} \frac{\widehat{\zeta_{0}}(k, \ell) e^{i(k x+\ell y)}}{\cosh (m h)} \cos \omega t d k d \ell, \\
\mathbf{u}(x, y, t ; 0) & =\frac{i g}{4 \pi^{2}} \iint_{\mathbb{R}^{2}} \frac{\mathbf{k} \widehat{\zeta_{0}}(k, \ell) e^{i(k x+\ell y)}}{\cosh (m h)} \frac{\sin \omega t}{\omega} d \mathbf{k}, \\
w(x, y, t ; 0) & =-\frac{1}{4 \pi^{2}} \iint_{\mathbb{R}^{2}} \frac{\widehat{\zeta_{0}}(k, \ell) e^{i(k x+\ell y)}}{\cosh (m h)} \omega \sin \omega t d \mathbf{k} .
\end{aligned}
$$

At time $t=0$, there is a singularity that can be incorporated in the above expressions. For simplicity, we only consider the expressions for $t>0$.

Since tsunameters have one component that measures the pressure at the bottom (see for example [11]), it is interesting to provide as well the expression $p_{b}(x, y, t)$ for the pressure at the bottom. The pressure $p(x, y, z, t)$ can be obtained from Bernoulli's equation, which was written explicitly for the free surface in equation (2), but is valid everywhere in the fluid:

$$
\frac{\partial \phi}{\partial t}+\frac{1}{2}|\nabla \phi|^{2}+g z+\frac{p}{\rho}=0
$$

After linearization, equation (28) becomes

$$
\frac{\partial \phi}{\partial t}+g z+\frac{p}{\rho}=0
$$

Along the bottom, it reduces to

$$
\frac{\partial \phi}{\partial t}+g(-h+\zeta)+\frac{p_{b}}{\rho}=0, \quad z=-h .
$$

The time-derivative of the velocity potential is readily available in Fourier space. Inverting the Fourier and Laplace transforms and evaluating the resulting expression at $z=-h$ gives for an instantaneous seabed deformation

$$
\left.\frac{\partial \phi}{\partial t}\right|_{z=-h}=-\frac{g}{(2 \pi)^{2}} \iint_{\mathbb{R}^{2}} \frac{\widehat{\zeta_{0}}(k, \ell) e^{i(k x+\ell y)}}{\cosh ^{2}(m h)} \cos \omega t d \mathbf{k} .
$$

The bottom pressure deviation from the hydrostatic pressure is then given by

$$
p_{b}(x, y, t)=-\left.\rho \frac{\partial \phi}{\partial t}\right|_{z=-h}-\rho g \zeta .
$$

Away from the deformed seabed, $\zeta$ goes to 0 so that $p_{b}$ simply is $-\left.\rho \phi_{t}\right|_{z=-h}$. The only difference between $p_{b}$ and $\rho g \eta$ is the presence of an additional $\cosh (m h)$ term in the denominator of $p_{b}$. 


\subsection{Passive generation}

In this case equation (11) becomes

$$
\frac{\partial \phi}{\partial z}=0, \quad z=-h
$$

and the initial condition for $\eta$ now reads

$$
\eta(x, y, 0)=\zeta_{0}(x, y)
$$

where $\zeta_{0}(x, y)$ is the seafloor deformation. Initial velocities are assumed to be zero.

Again we apply the Fourier transform in the horizontal coordinates $(x, y)$. The Laplace transform is not applied since there is no substantial dynamics in the problem. Equations (9), (31) and (13) become

$$
\begin{gathered}
\frac{d^{2} \widehat{\phi}}{d z^{2}}-\left(k^{2}+\ell^{2}\right) \widehat{\phi}=0, \\
\frac{d \widehat{\phi}}{d z}(k, \ell,-h, t)=0, \\
\frac{\partial^{2} \widehat{\phi}}{\partial t^{2}}(k, \ell, 0, t)+g \frac{\partial \widehat{\phi}}{\partial z}(k, \ell, 0, t)=0 .
\end{gathered}
$$

A general solution to Laplace's equation (32) is again given by

$$
\widehat{\phi}(k, \ell, z, t)=A(k, \ell, t) \cosh (m z)+B(k, \ell, t) \sinh (m z),
$$

where $m=\sqrt{k^{2}+\ell^{2}}$. The relationship between the functions $A(k, \ell, t)$ and $B(k, \ell, t)$ can be easily found from the boundary condition (33):

$$
B(k, \ell, t)=A(k, \ell, t) \tanh (m h) .
$$

From equation (34) and the initial conditions one finds

$$
A(k, \ell, t)=-\frac{g}{\omega} \widehat{\zeta_{0}}(k, \ell) \sin \omega t
$$

Substituting the expressions for the functions $A$ and $B$ in (35) yields

$$
\widehat{\phi}(k, \ell, z, t)=-\frac{g}{\omega} \widehat{\zeta}_{0}(k, \ell) \sin \omega t(\cosh (m z)+\tanh (m h) \sinh (m z)) .
$$

From (12), the free-surface elevation becomes

$$
\widehat{\eta}(k, \ell, t)=\widehat{\zeta_{0}}(k, \ell) \cos \omega t .
$$

Inverting the Fourier transform provides the general integral solution

$$
\eta(x, y, t)=\frac{1}{(2 \pi)^{2}} \iint_{\mathbb{R}^{2}} \widehat{\zeta_{0}}(k, \ell) \cos \omega t e^{i(k x+\ell y)} d k d \ell .
$$


Let us now evaluate the velocity field in the fluid domain. Equation (38) gives the Fourier transform of the velocity potential $\phi(x, y, z, t)$. Taking the Fourier transform of

$$
\mathbf{u}(x, y, t ; \beta)=\left.\nabla_{h} \phi(x, y, z, t)\right|_{z=\beta h}
$$

yields

$$
\begin{aligned}
\widehat{\mathbf{u}}(k, \ell, t ; \beta) & =-i \widehat{\phi}(k, \ell, \beta h, t) \mathbf{k} \\
& =i \frac{g}{\omega} \widehat{\zeta_{0}}(k, \ell) \sin \omega t(\cosh (\beta m h)+\tanh (m h) \sinh (\beta m h)) \mathbf{k} .
\end{aligned}
$$

Inverting the Fourier transform gives the general formula for the horizontal velocities

$\mathbf{u}(x, y, t ; \beta)=\frac{i g}{4 \pi^{2}} \iint_{\mathbb{R}^{2}} \mathbf{k} \widehat{\zeta_{0}}(k, \ell) \frac{\sin \omega t}{\omega}(\cosh (\beta m h)+\tanh (m h) \sinh (\beta m h)) e^{i(k x+\ell y)} d \mathbf{k}$.

Along the free surface $\beta=0$, the horizontal velocity vector becomes

$$
\mathbf{u}(x, y, t ; 0)=\frac{i g}{4 \pi^{2}} \iint_{\mathbb{R}^{2}} \mathbf{k} \widehat{\zeta_{0}}(k, \ell) \frac{\sin \omega t}{\omega} e^{i(k x+\ell y)} d \mathbf{k} .
$$

Next we determine the vertical component of the velocity $w(x, y, t ; \beta)$ at a given vertical level $z=\beta h$. It is easy to obtain the Fourier transform $\widehat{w}(k, \ell, t ; \beta)$ by differentiating (38):

$$
\widehat{w}(k, \ell, t ; \beta)=\left.\frac{\partial \widehat{\phi}}{\partial z}\right|_{z=\beta h}=-m g \frac{\sin \omega t}{\omega} \widehat{\zeta_{0}}(k, \ell)(\sinh (\beta m h)+\tanh (m h) \cosh (\beta m h)) .
$$

Inverting this transform yields

$$
\begin{aligned}
& w(x, y, t ; \beta)=-\frac{g}{4 \pi^{2}} \iint_{\mathbb{R}^{2}} \frac{m \sin \omega t}{\omega} \widehat{\zeta_{0}}(k, \ell)(\sinh (\beta m h)+ \\
&\tanh (m h) \cosh (\beta m h)) e^{i(k x+\ell y)} d \mathbf{k}
\end{aligned}
$$

for $-1 \leq \beta \leq 0$. Using the dispersion relation, one can write the vertical component of the velocity along the free surface $(\beta=0)$ as

$$
w(x, y, t ; 0)=-\frac{1}{4 \pi^{2}} \iint_{\mathbb{R}^{2}} \omega \sin \omega t \widehat{\zeta_{0}}(k, \ell) e^{i(k x+\ell y)} d \mathbf{k} .
$$

All the formulas obtained in this section are valid only if the integrals converge.

Again, one can compute the bottom pressure. At $z=-h$, one has

$$
\left.\frac{\partial \phi}{\partial t}\right|_{z=-h}=-\frac{g}{(2 \pi)^{2}} \iint_{\mathbb{R}^{2}} \frac{\widehat{\zeta_{0}}(k, \ell) e^{i(k x+\ell y)}}{\cosh (m h)} \cos \omega t d \mathbf{k}
$$


The bottom pressure deviation from the hydrostatic pressure is then given by

$$
p_{b}(x, y, t)=-\left.\rho \frac{\partial \phi}{\partial t}\right|_{z=-h}-\rho g \zeta .
$$

Again, away from the deformed seabed, $p_{b}$ reduces to $-\left.\rho \phi_{t}\right|_{z=-h}$. The only difference between $p_{b}$ and $\rho g \eta$ is the presence of an additional $\cosh (m h)$ term in the denominator of $p_{b}$.

The main differences between passive and active generation processes are that (i) the wave amplitudes and velocities obtained with the instantly moving bottom are lower than those generated by initial translation of the bottom motion and that (ii) the water column plays the role of a low-pass filter (compare equations (25)-(27) with equations (39)-(41)). High frequencies are attenuated in the moving bottom solution. Ward [36], who studied landslide tsunamis, also commented on the $1 / \cosh (m h)$ term, which low-pass filters the source spectrum. So the filter favors long waves. In the discussion section, we will come back to the differences between passive generation and active generation.

\subsection{Linear numerical method}

All the expressions derived from linear theory are explicit but they must be computed numerically. It is not a trivial task because of the oscillatory behaviour of the integrand functions. All integrals were computed with Filon type numerical integration formulas [6], which explicitly take into account this oscillatory behaviour. Numerical results will be shown in Section 6 .

\section{Nonlinear shallow water equations}

Synolakis and Bernard [28] introduced a clear distinction between the various shallow-water models. At the lowest order of approximation, one obtains the linear shallow water wave equation. The next level of approximation provides the nondispersive nonlinear shallow water equations (NSW). In the next level, dispersive terms are added and the resulting equations constitute the Boussinesq equations. Since there are many different ways to go to this level of approximation, there are a lot of different types of Boussinesq equations. The NSW equations are the most commonly used equations for tsunami propagation (see in particular the code MOST developed by the National Oceanic and Atmospheric Administration in the US [29] or the code TUNAMI developed by the Disaster Control Research Center in Japan). They are also used for generation and runup/inundation. For wave runup, the effects of bottom friction become important and must be included in the codes. Our analysis will focus on the NSW equations. For simplicity, we assume below that $h$ is constant. Therefore one can take $h$ as reference depth, so that the seafloor is given by $z=-1+\varepsilon \zeta$. 


\subsection{Mathematical model}

In this subsection, partial derivatives are denoted by subscripts. When $\mu^{2}$ is a small parameter, the water is considered to be shallow. For the shallow water theory, one formally expands the potential $\phi$ in powers of $\mu^{2}$ :

$$
\phi=\phi_{0}+\mu^{2} \phi_{1}+\mu^{4} \phi_{2}+\cdots .
$$

This expansion is substituted into the governing equation and the boundary conditions. The lowest-order term in Laplace's equation is

$$
\phi_{0 z z}=0 .
$$

The boundary conditions imply that $\phi_{0}=\phi_{0}(x, y, t)$. Thus the vertical velocity component is zero and the horizontal velocity components are independent of the vertical coordinate $z$ at lowest order. Let us introduce the notation $u:=$ $\phi_{0 x}(x, y, t)$ and $v:=\phi_{0 y}(x, y, t)$. Solving Laplace's equation and taking into account the bottom kinematic condition yield the following expressions for $\phi_{1}$ and $\phi_{2}$ :

$$
\begin{aligned}
\phi_{1}(x, y, z, t)= & -\frac{1}{2} Z^{2}\left(u_{x}+v_{y}\right)+z\left[\zeta_{t}+\varepsilon\left(u \zeta_{x}+v \zeta_{y}\right)\right], \\
\phi_{2}(x, y, z, t)= & \frac{1}{24} Z^{4}\left(\Delta u_{x}+\Delta v_{y}\right)+\varepsilon\left(\varepsilon \frac{z^{2}}{2}|\nabla \zeta|^{2}-\frac{1}{6} Z^{3} \Delta \zeta\right)\left(u_{x}+v_{y}\right) \\
& -\frac{\varepsilon}{3} Z^{3} \nabla \zeta \cdot \nabla\left(u_{x}+v_{y}\right)-\frac{z^{3}}{6}\left(\Delta \zeta_{t}+\varepsilon \Delta\left(u \zeta_{x}+v \zeta_{y}\right)\right)+ \\
& z(-1+\varepsilon \zeta)\left[\varepsilon \nabla \zeta \cdot \nabla\left(\zeta_{t}+\varepsilon\left(u \zeta_{x}+v \zeta_{y}\right)\right)-\varepsilon^{2}|\nabla \zeta|^{2}\left(u_{x}+v_{y}\right)-\right. \\
& \left.\frac{1}{2}(-1+\varepsilon \zeta)\left(\Delta \zeta_{t}+\varepsilon \Delta\left(u \zeta_{x}+v \zeta_{y}\right)\right)\right],
\end{aligned}
$$

where

$$
Z=1+z-\varepsilon \zeta
$$

The next step consists in retaining terms of requested order in the freesurface boundary conditions. Powers of $\varepsilon$ will appear when expanding in Taylor series the free-surface conditions around $z=0$. For example, if one keeps terms of order $\varepsilon \mu^{2}$ and $\mu^{4}$ in the dynamic boundary condition (6) and in the kinematic boundary condition (4), one obtains

$$
\begin{array}{r}
\mu^{2} \phi_{0 t}-\frac{1}{2} \mu^{4}\left(u_{t x}+v_{t y}\right)+\mu^{2} \eta+\frac{1}{2} \varepsilon \mu^{2}\left(u^{2}+v^{2}\right)=0 \\
\mu^{2}\left[\eta_{t}+\varepsilon\left(u \eta_{x}+v \eta_{y}\right)+(1+\varepsilon(\eta-\zeta))\left(u_{x}+v_{y}\right)-\zeta_{t}-\varepsilon\left(u \zeta_{x}+v \zeta_{y}\right)\right]= \\
\frac{1}{6} \mu^{4}\left(\Delta u_{x}+\Delta v_{y}\right) .
\end{array}
$$


Differentiating (43) first with respect to $x$ and then with respect to $y$ gives a set of two equations:

$$
\begin{aligned}
& u_{t}+\varepsilon\left(u u_{x}+v v_{x}\right)+\eta_{x}-\frac{1}{2} \mu^{2}\left(u_{t x x}+v_{t x y}\right)=0 \\
& v_{t}+\varepsilon\left(u u_{y}+v v_{y}\right)+\eta_{y}-\frac{1}{2} \mu^{2}\left(u_{t x y}+v_{t y y}\right)=0 .
\end{aligned}
$$

The kinematic condition (44) becomes

$$
(\eta-\zeta)_{t}+[u(1+\varepsilon(\eta-\zeta))]_{x}+[v(1+\varepsilon(\eta-\zeta))]_{y}=\frac{1}{6} \mu^{2}\left(\Delta u_{x}+\Delta v_{y}\right) .
$$

Equations (45),(46) and (47) contain in fact various shallow-water models. The so-called fundamental NSW equations which contain no dispersive effects are obtained by neglecting the terms of order $\mu^{2}$ :

$$
\begin{aligned}
u_{t}+\varepsilon\left(u u_{x}+v u_{y}\right)+\eta_{x} & =0, \\
v_{t}+\varepsilon\left(u v_{x}+v v_{y}\right)+\eta_{y} & =0, \\
\eta_{t}+[u(1+\varepsilon(\eta-\zeta))]_{x}+[v(1+\varepsilon(\eta-\zeta))]_{y} & =\zeta_{t} .
\end{aligned}
$$

Going back to a bathymetry $h^{*}(x, y, t)$ equal to $1-\varepsilon \zeta(x, y, t)$ and using the fact that $(u, v)$ is the horizontal gradient of $\phi_{0}$, one can rewrite the system of NSW equations as

$$
\begin{aligned}
u_{t}+\frac{\varepsilon}{2}\left(u^{2}+v^{2}\right)_{x}+\eta_{x} & =0, \\
v_{t}+\frac{\varepsilon}{2}\left(u^{2}+v^{2}\right)_{y}+\eta_{y} & =0, \\
\eta_{t}+\left[u\left(h^{*}+\varepsilon \eta\right)\right]_{x}+\left[v\left(h^{*}+\varepsilon \eta\right)\right]_{y} & =-\frac{1}{\varepsilon} h_{t}^{*} .
\end{aligned}
$$

The system of equations (51)-(53) has been used for example by Titov and Synolakis [29] for the numerical computation of tidal wave run-up. Note that this model does not include any bottom friction terms.

The NSW equations with dispersion (45)-(47), also known as the Boussinesq equations, can be written in the following form:

$$
\begin{aligned}
u_{t}+\frac{\varepsilon}{2}\left(u^{2}+v^{2}\right)_{x}+\eta_{x}-\frac{1}{2} \mu^{2} \Delta u_{t} & =0, \\
v_{t}+\frac{\varepsilon}{2}\left(u^{2}+v^{2}\right)_{y}+\eta_{y}-\frac{1}{2} \mu^{2} \Delta v_{t} & =0, \\
\eta_{t}+\left[u\left(h^{*}+\varepsilon \eta\right)\right]_{x}+\left[v\left(h^{*}+\varepsilon \eta\right)\right]_{y}-\frac{1}{6} \mu^{2}\left(\Delta u_{x}+\Delta v_{y}\right) & =-\frac{1}{\varepsilon} h_{t}^{*} .
\end{aligned}
$$

Kulikov et al. [21] have argued that the satellite altimetry observations of the Indian Ocean tsunami show some dispersive effects. However the steepness is so small that the origin of these effects is questionable. Guesmia et al. [14] compared Boussinesq and shallow-water models and came to the conclusion that 
the effects of frequency dispersion are minor. As pointed out in [19], dispersive effects are necessary only when examining steep gravity waves, which are not encountered in the context of tsunami hydrodynamics in deep water. However they can be encountered in experiments such as those of Hammack [15] because the parameter $\mu$ is much bigger.

\subsection{Numerical method}

In order to solve the NSW equations, a finite-volume approach is used. For example LeVeque [23] used a high-order finite volume scheme to solve a system of NSW equations. Here the flux scheme we use is the characteristic flux scheme, which was introduced by Ghidaglia et al. [9]. This numerical method satisfies the conservative properties at the discrete level. The NSW equations (51)-(53) can be rewritten in the following conservative form:

$$
\frac{\partial \mathbf{w}}{\partial t}+\frac{\partial \mathbf{F}(\mathbf{w})}{\partial x}+\frac{\partial \mathbf{G}(\mathbf{w})}{\partial y}=\mathbf{S}(x, y, \mathbf{w}, t)
$$

where

$$
\begin{aligned}
\mathbf{w} & =(\eta, u, v), \\
\mathbf{F} & =\left(u\left(h^{*}+\varepsilon \eta\right), \frac{\varepsilon}{2}\left(u^{2}+v^{2}\right)+\eta, 0\right), \\
\mathbf{G} & =\left(v\left(h^{*}+\varepsilon \eta\right), 0, \frac{\varepsilon}{2}\left(u^{2}+v^{2}\right)+\eta\right), \\
\mathbf{S} & =\left(-\zeta_{t}, 0,0\right) .
\end{aligned}
$$

The scheme we use is multi-dimensional by construction and does not require the solution of any Riemann problem. For the sake of simplicity in the description of the numerical method, we assume that there is no $y$-variation and no source term $\mathbf{S}$. Let us then consider a system of $m$-conservation laws $(m \geq 1)$

$$
\frac{\partial \mathbf{w}}{\partial t}+\frac{\partial \mathbf{F}(\mathbf{w})}{\partial x}=0, \quad x \in \mathbb{R}, \quad t \geq 0,
$$

where $\mathbf{w} \in \mathbb{R}^{m}$ and $\mathbf{F}: \mathbb{R}^{m} \mapsto \mathbb{R}^{m}$. We denote by $\mathbf{A}(\mathbf{w})$ the Jacobian matrix of $\mathbf{F}(\mathbf{w})$ :

$$
\mathbf{A}_{i j}(\mathbf{w})=\frac{\partial \mathbf{F}_{i}}{\partial \mathbf{w}_{j}}(\mathbf{w}), \quad 1 \leq i, j \leq m .
$$

The system (62) is assumed to be hyperbolic. In other words, for every $\mathbf{w}$ there exists a smooth basis $\left(r_{1}(\mathbf{w}), \ldots, r_{m}(\mathbf{w})\right)$ of $\mathbb{R}^{m}$ consisting of eigenvectors of $\mathbf{A}(\mathbf{w})$. Said differently, there exists $\lambda_{k}(\mathbf{w}) \in \mathbb{R}$ such that $\mathbf{A}(\mathbf{w}) r_{k}(\mathbf{w})=$ $\lambda_{k}(\mathbf{w}) r_{k}(\mathbf{w})$. It is then possible to construct $\left(\ell_{1}(\mathbf{w}), \ldots, \ell_{m}(\mathbf{w})\right)$ such that

$$
{ }^{t} \mathbf{A}(\mathbf{w}) \ell_{k}(\mathbf{w})=\lambda_{k}(\mathbf{w}) \ell_{k}(\mathbf{w}) \text { and } \ell_{k}(\mathbf{w}) \cdot r_{p}(\mathbf{w})=\delta_{k p} .
$$

Let $\mathbb{R}=\cup_{j \in \mathbb{Z}}\left[x_{j-1 / 2}, x_{j+1 / 2}\right]$ be a one-dimensional mesh. Let also $\mathbb{R}_{+}=$ $\cup_{n \in \mathbb{N}}\left[t_{n}, t_{n+1}\right]$. Let us discretize (62) by a finite volume method. We set

$$
\Delta x_{j}=x_{j+1 / 2}-x_{j-1 / 2}, \quad \Delta t_{n}=t_{n+1}-t_{n}
$$


and

$$
\widetilde{\mathbf{w}}_{j}^{n}=\frac{1}{\Delta x_{j}} \int_{x_{j-1 / 2}}^{x_{j+1 / 2}} \mathbf{w}\left(x, t_{n}\right) d x, \quad \widetilde{\mathbf{F}}_{j+1 / 2}^{n}=\frac{1}{\Delta t_{n}} \int_{t_{n}}^{t_{n+1}} \mathbf{F}\left(\mathbf{w}\left(x_{j+1 / 2}, t\right)\right) d t .
$$

The system (62) can then be rewritten (exactly) as

$$
\widetilde{\mathbf{w}}_{j}^{n+1}=\widetilde{\mathbf{w}}_{j}^{n}-\frac{\Delta t_{n}}{\Delta x_{j}}\left(\widetilde{\mathbf{F}}_{j+1 / 2}^{n}-\widetilde{\mathbf{F}}_{j-1 / 2}^{n}\right) .
$$

For a three-point explicit numerical scheme one has

$$
\widetilde{\mathbf{F}}_{j+1 / 2}^{n} \approx \mathbf{f}_{j}^{n}\left(\mathbf{w}_{j}^{n}, \mathbf{w}_{j+1}^{n}\right),
$$

where the function $\mathbf{f}$ is to be specified. Multiplying (62) by $\mathbf{A}(\mathbf{w})$ yields

$$
\frac{\partial \mathbf{F}(\mathbf{w})}{\partial t}+\mathbf{A}(\mathbf{w}) \frac{\partial \mathbf{F}(\mathbf{w})}{\partial x}=0 .
$$

This shows that the flux $\mathbf{F}(\mathbf{w})$ is advected by $\mathbf{A}(\mathbf{w})$. The numerical flux $\mathbf{f}_{j}^{n}\left(\mathbf{w}_{j}^{n}, \mathbf{w}_{j+1}^{n}\right)$ represents the flux at an interface. Using a mean value $\mu_{j+1 / 2}^{n}$ of $\mathbf{w}$ at this interface, we replace (66) by the linearization

$$
\frac{\partial \mathbf{F}(\mathbf{w})}{\partial t}+\mathbf{A}\left(\mu_{j+1 / 2}^{n}\right) \frac{\partial \mathbf{F}(\mathbf{w})}{\partial x}=0 .
$$

We define the $k$-th characteristic flux component to be $F_{k}(\mathbf{w})=\ell_{k}\left(\mu_{j+1 / 2}^{n}\right)$. $\mathbf{F}(\mathbf{w})$. It follows that

$$
\frac{\partial F_{k}(\mathbf{w})}{\partial t}+\lambda_{k}\left(\mu_{j+1 / 2}^{n}\right) \frac{\partial F_{k}(\mathbf{w})}{\partial x}=0 .
$$

This linear equation can be solved explicitly for $F_{k}(\mathbf{w})$. As a result it is natural to define the characteristic flux $\mathbf{f}^{C F}$ at the interface between two cells $\left[x_{j-1 / 2}, x_{j+1 / 2}\right]$ and $\left[x_{j+1 / 2}, x_{j+3 / 2}\right]$ as follows: for $k \in 1, \ldots, m$,

$\ell_{k}\left(\mu_{j+1 / 2}^{n}\right) \cdot \mathbf{f}_{j}^{C F, n}\left(\mathbf{w}_{j}^{n}, \mathbf{w}_{j+1}^{n}\right)=\ell_{k}\left(\mu_{j+1 / 2}^{n}\right) \cdot \mathbf{F}\left(\mathbf{w}_{j}^{n}\right)$, when $\lambda_{k}\left(\mu_{j+1 / 2}^{n}\right)>0$,

$\ell_{k}\left(\mu_{j+1 / 2}^{n}\right) \cdot \mathbf{f}_{j}^{C F, n}\left(\mathbf{w}_{j}^{n}, \mathbf{w}_{j+1}^{n}\right)=\ell_{k}\left(\mu_{j+1 / 2}^{n}\right) \cdot \mathbf{F}\left(\mathbf{w}_{j+1}^{n}\right)$, when $\lambda_{k}\left(\mu_{j+1 / 2}^{n}\right)<0$,

$\ell_{k}\left(\mu_{j+1 / 2}^{n}\right) \cdot \mathbf{f}_{j}^{C F, n}\left(\mathbf{w}_{j}^{n}, \mathbf{w}_{j+1}^{n}\right)=\ell_{k}\left(\mu_{j+1 / 2}^{n}\right) \cdot\left(\frac{\mathbf{F}\left(\mathbf{w}_{j}^{n}\right)+\mathbf{F}\left(\mathbf{w}_{j+1}^{n}\right)}{2}\right)$,

when $\lambda_{k}\left(\mu_{j+1 / 2}^{n}\right)=0$. Here

$$
\mu_{j+1 / 2}^{n}=\frac{\Delta x_{j} \mathbf{w}_{j}^{n}+\Delta x_{j+1} \mathbf{w}_{j+1}^{n}}{\Delta x_{j}+\Delta x_{j+1}} .
$$

The characteristic flux can be written as

$$
\mathbf{f}_{j}^{C F, n}\left(\mathbf{w}_{j}^{n}, \mathbf{w}_{j+1}^{n}\right)=\mathbf{f}^{C F}\left(\mu_{j}^{n} ; \mathbf{w}_{j}^{n}, \mathbf{w}_{j+1}^{n}\right)
$$


where

$$
\mathbf{f}^{C F}\left(\mu ; \mathbf{w}_{1}, \mathbf{w}_{2}\right)=\frac{\mathbf{F}\left(\mathbf{w}_{1}\right)+\mathbf{F}\left(\mathbf{w}_{2}\right)}{2}-\operatorname{sgn}\left(\mathbf{A}\left(\mu\left(\mathbf{w}_{1}, \mathbf{w}_{2}\right)\right) \frac{\mathbf{F}\left(\mathbf{w}_{2}\right)-\mathbf{F}\left(\mathbf{w}_{1}\right)}{2} .\right.
$$

The sign of the matrix $\mathbf{A}(\mu)$ is defined by

$$
\operatorname{sgn}(\mathbf{A}(\mu)) \Phi=\sum_{k=1}^{k=m} \operatorname{sgn}\left(\lambda_{k}\right)\left(\ell_{k}(\mu) \cdot \Phi\right) r_{k}(\mu) .
$$

Going back to (64), one can construct the following explicit scheme:

$$
\mathbf{w}_{j}^{n+1}=\mathbf{w}_{j}^{n}-\frac{\Delta t_{n}}{\Delta x_{j}}\left(\mathbf{f}_{j}^{C F, n}\left(\mathbf{w}_{j}^{n}, \mathbf{w}_{j+1}^{n}\right)-\mathbf{f}_{j}^{C F, n}\left(\mathbf{w}_{j-1}^{n}, \mathbf{w}_{j}^{n}\right)\right) .
$$

The characteristic flux scheme (69) gives very good results, especially when complex systems are considered [9]. In our case, we have to consider equation (62) in two dimensions and to discretise the source term too:

$$
\frac{\partial \mathbf{w}}{\partial t}+\frac{\partial \mathbf{F}(\mathbf{w})}{\partial x}+\frac{\partial \mathbf{G}(\mathbf{w})}{\partial y}=\mathbf{S}(x, y, \mathbf{w}, t) .
$$

One can refer to [10] for these two extensions.

\section{$5 \quad$ Numerical method for the full equations}

The fully nonlinear potential flow (FNPF) equations (3)-(6) are solved by using a numerical model based on the Boundary Element Method (BEM). An accurate code was developed by Grilli et al. [12]. It uses a high-order three-dimensional boundary element method combined with mixed Eulerian-Lagrangian time updating, based on second-order explicit Taylor expansions with adaptive time steps. The efficiency of the code was recently greatly improved by introducing a more efficient spatial solver, based on the fast multipole algorithm [7]. By replacing every matrix-vector product of the iterative solver and avoiding the building of the influence matrix, this algorithm reduces the computing complexity from $O\left(N^{2}\right)$ to nearly $O(N)$ up to logarithms, where $N$ is the number of nodes on the boundary.

By using Green's second identity, Laplace's equation (1) is transformed into the boundary integral equation

$$
\alpha\left(\mathbf{x}_{l}\right) \phi\left(\mathbf{x}_{l}\right)=\int_{\Gamma}\left(\frac{\partial \phi}{\partial n}(\mathbf{x}) G\left(\mathbf{x}, \mathbf{x}_{l}\right)-\phi(\mathbf{x}) \frac{\partial G}{\partial n}\left(\mathbf{x}, \mathbf{x}_{l}\right)\right) d \Gamma
$$

where $G$ is the three-dimensional free space Green's function. The notation $\partial G / \partial n$ means the normal derivative, that is $\partial G / \partial n=\nabla G \cdot \mathbf{n}$, with $\mathbf{n}$ the unit outward normal vector. The vectors $\mathbf{x}=(x, y, z)$ and $\mathbf{x}_{l}=\left(x_{l}, y_{l}, z_{l}\right)$ are position vectors for points on the boundary, and $\alpha\left(\mathbf{x}_{l}\right)=\theta_{l} /(4 \pi)$ is a geometric 
coefficient, with $\theta_{l}$ the exterior solid angle made by the boundary at point $\mathbf{x}_{l}$. The boundary $\Gamma$ is divided into various parts with different boundary conditions. On the free surface, one rewrites the nonlinear kinematic and dynamic boundary conditions in a mixed Eulerian-Lagrangian form,

$$
\begin{aligned}
\frac{D \mathbf{R}}{D t} & =\nabla \phi, \\
\frac{D \phi}{D t} & =-g z+\frac{1}{2} \nabla \phi \cdot \nabla \phi,
\end{aligned}
$$

with $\mathbf{R}$ the position vector of a free-surface fluid particle. The material derivative is defined as

$$
\frac{D}{D t}=\frac{\partial}{\partial t}+\mathbf{q} \cdot \nabla
$$

For time integration, second-order explicit Taylor series expansions are used to find the new position and the potential on the free surface at time $t+\Delta t$. This time stepping scheme presents the advantage of being explicit, and the use of spatial derivatives along the free surface provides a better stability of the computed solution.

The integral equations are solved by BEM. The boundary is discretized into $N$ collocation nodes and $M$ high-order elements are used to interpolate between these nodes. Within each element, the boundary geometry and the field variables are discretized using polynomial shape functions. The integrals on the boundary are converted into a sum on the elements, each one being calculated on the reference element. The matrices are built with the numerical computation of the integrals on the reference element. The linear systems resulting from the two boundary integral equations (one for the pair $(\phi, \partial \phi / \partial n)$ and one for the pair $\left.\left(\partial \phi / \partial t, \partial^{2} \phi / \partial t \partial n\right)\right)$ are full and non symmetric. Assembling the matrix as well as performing the integrations accurately are time consuming tasks. They are done only once at each time step, since the same matrix is used for both systems. Solving the linear system is another time consuming task. Even with the GMRES algorithm with preconditioning, the computational complexity is $O\left(N^{2}\right)$, which is the same as the complexity of the assembling phase. The introduction of the fast multipole algorithm reduces considerably the complexity of the problem. The matrix is no longer built. Far away nodes are placed in groups, so less time is spent in numerical integrations and memory requirements are reduced. The hierarchical structure involved in the algorithm gives automatically the distance criteria for adaptive integrations.

Grilli et al. [13] used the earlier version of the code to study tsunami generation by underwater landslides. They included the bottom motion due to the landslide. For the comparisons shown below, we only used the passive approach: we did not include the dynamics of the bottom motion.

\section{Comparisons and discussion}

The passive generation approach is followed for the numerical comparisons between the three models: (i) linear equations, (ii) NSW equations and (iii) fully 
nonlinear equations. As shown in Section 3, this generation process gives the largest transient-wave amplitudes for a given permanent deformation of the seafloor. Therefore it is in some sense a worst case scenario.

The small dimensionless numbers $\varepsilon$ and $\mu^{2}$ introduced in (7) represent the magnitude of the nonlinear terms and dispersive terms in the governing equations, respectively. Hence, the relative importance of the nonlinear and the dispersive effects is given by the parameter

$$
S=\frac{\text { nonlinear terms }}{\text { dispersive terms }}=\frac{\varepsilon}{\mu^{2}}=\frac{a \lambda^{2}}{d^{3}},
$$

which is called the Stokes (or Ursell) number [34]. ${ }^{5}$ An important assumption in the derivation of the Boussinesq system (54)-(56) is that the Ursell number is $O(1)$. Here, the symbol $O(\cdot)$ is used informally in the way that is common in the construction and formal analysis of model equations for physical phenomena. We are concerned with the limits $\varepsilon \rightarrow 0$ and $\mu \rightarrow 0$. Thus, $S=O(1)$ means that, as $\varepsilon \rightarrow 0$ and $\mu \rightarrow 0, S$ takes values that are neither very large nor very small. We emphasize here that the Ursell number does not convey any information by itself about the separate negligibility of nonlinear and frequency dispersion effects. Another important aspect of models is the time scale of their validity. In the NSW equations, terms of order $O\left(\varepsilon^{2}\right)$ and $O\left(\mu^{2}\right)$ have been neglected. Therefore one expects these terms to make an order-one relative contribution on a time scale of order $\min \left(\varepsilon^{-2}, \mu^{-2}\right)$.

All the figures shown below are two-dimensional plots for convenience but we recall that all computations for the three models are three-dimensional. Figure 6 shows profiles of the free-surface elevation along the main direction of propagation ( $y$-axis) of transient waves generated by a permanent seafloor deformation corresponding to the parameters given in Table 1. This deformation, which has been plotted in figure 2, has been translated to the free surface. The water depth is $100 \mathrm{~m}$. The small dimensionless numbers are roughly $\varepsilon=5 \times 10^{-4}$ and $\mu=10^{-2}$, with a corresponding Ursell number equal to 5 . One can see that the front system splits in two and propagates in both directions, with a leading wave of depression to the left and a leading wave of elevation to the right, in qualitative agreement with the satellite and tide gauge measurements of the 2004 Sumatra event. When tsunamis are generated along subduction zones, they usually split in two; one moves quickly inland while the second heads toward the open ocean. The three models are almost undistinguishable at all times: the waves propagate with the same speed and the same profile. Nonlinear effects and dispersive effects are clearly negligible during the first moments of transient

\footnotetext{
${ }^{5}$ One finds sometimes in the literature a subtle difference between the Stokes and Ursell numbers. Both involve a wave amplitude multiplied by the square of a wavelength divided by the cube of a water depth. The Stokes number is defined specifically for the excitation of a closed basin while the Ursell number is used in a more general context to describe the evolution of a long wave system. Therefore only the characteristic length is different. For the Stokes number the length is the usual wavelength $\lambda$ related to the frequency $\omega$ by $\lambda \approx 2 \pi \sqrt{g d} / \omega$. In the Ursell number, the length refers to the local wave shape independent of the exciting conditions.
} 

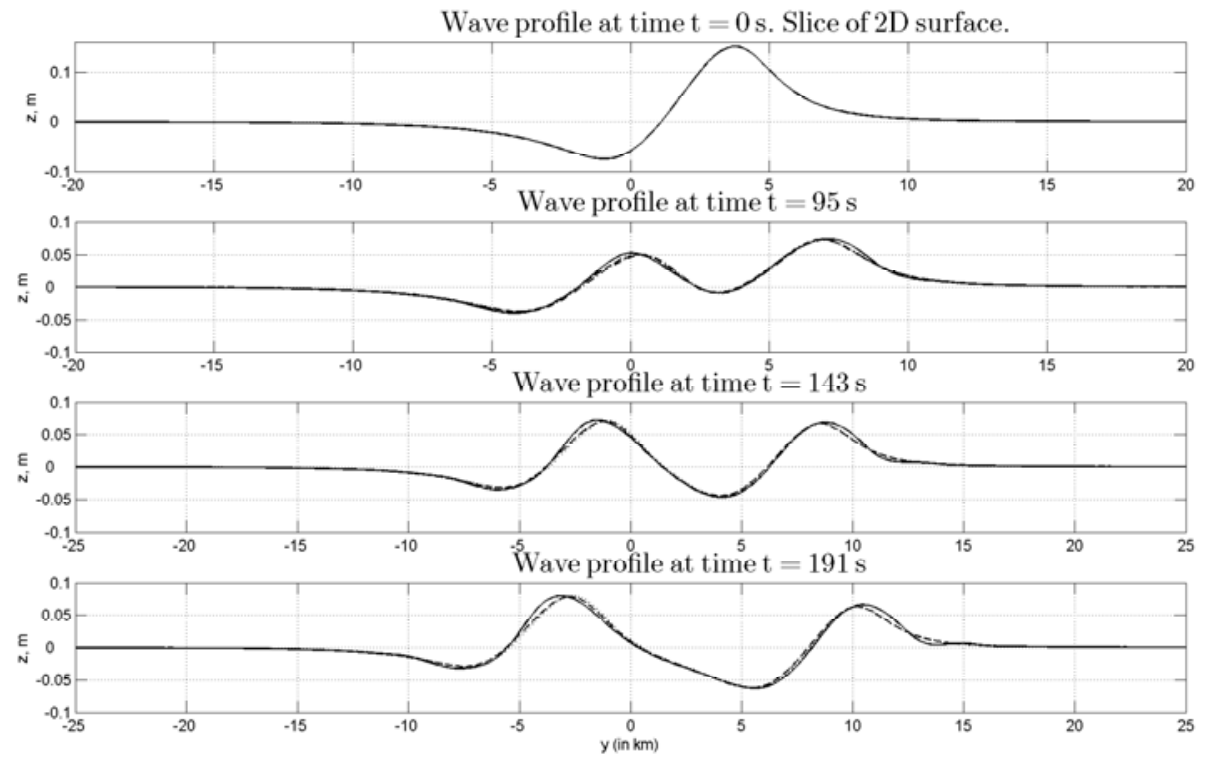

Figure 6: Comparisons of the free-surface elevation at $x=0$ resulting from the integration of the linear equations $(\cdots)$, NSW equations (--) and nonlinear equations (-) at different times of the propagation of transient waves generated by an earthquake $(t=0 \mathrm{~s}, t=95 \mathrm{~s}, t=143 \mathrm{~s}, t=191 \mathrm{~s})$. The parameters for the earthquake are those given in Table 1 . The water depth is $h=100 \mathrm{~m}$. One has the following estimates: $\varepsilon=5 \times 10^{-4}, \mu^{2}=10^{-4}$ and consequently $S=5$. 

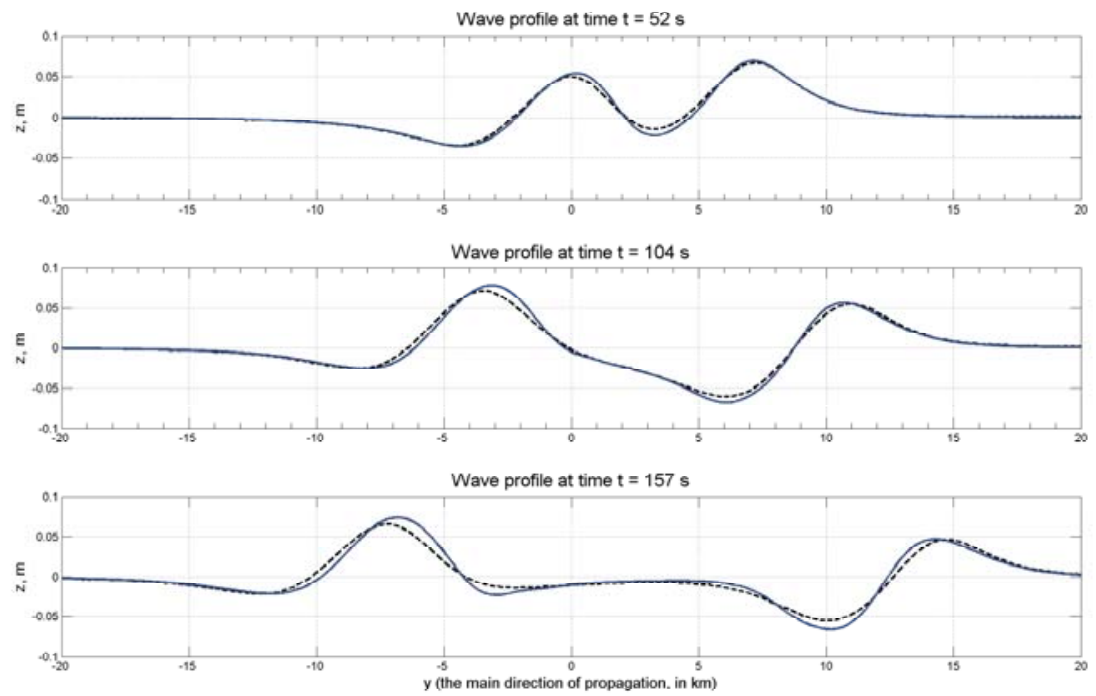

Figure 7: Comparisons of the free-surface elevation at $x=0$ resulting from the integration of the linear equations $(\cdots)$, NSW equations $(--)$ and nonlinear equations $(-)$ at different times of the propagation of transient waves generated by an earthquake $(t=52 \mathrm{~s}, t=104 \mathrm{~s}, t=157 \mathrm{~s})$. The parameters for the earthquake are those given in Table 1 . The water depth is $h=500 \mathrm{~m}$. One has the following estimates: $\varepsilon=10^{-4}, \mu^{2}=2.5 \times 10^{-3}$ and consequently $S=0.04$.

waves generated by a moving bottom, at least for these particular choices of $\varepsilon$ and $\mu$.

Let us now decrease the Ursell number by increasing the water depth. Figure 7 illustrates the evolution of transient water waves computed with the three models for the same parameters as those of figure 6 , except for the water depth now equal to $500 \mathrm{~m}$. The small dimensionless numbers are roughly $\varepsilon=10^{-4}$ and $\mu=5 \times 10^{-2}$, with a corresponding Ursell number equal to 0.04 . The linear and nonlinear profiles cannot be distinguished within graphical accuracy. Only the NSW profile is slightly different.

Let us introduce several sensors (tide gauges) at selected locations which are representative of the initial deformation of the free surface (see figure 8). One can study the evolution of the surface elevation during the generation time at each gauge. Figure 9 shows free-surface elevations corresponding to the linear and nonlinear shallow water models. They are plotted on the same graph for comparison purposes. Again there is a slight difference between the linear and the NSW models, but dispersion effects are still small.

Let us decrease the Ursell number even further by increasing the water depth. Figures 10 and 11 illustrate the evolution of transient water waves computed 


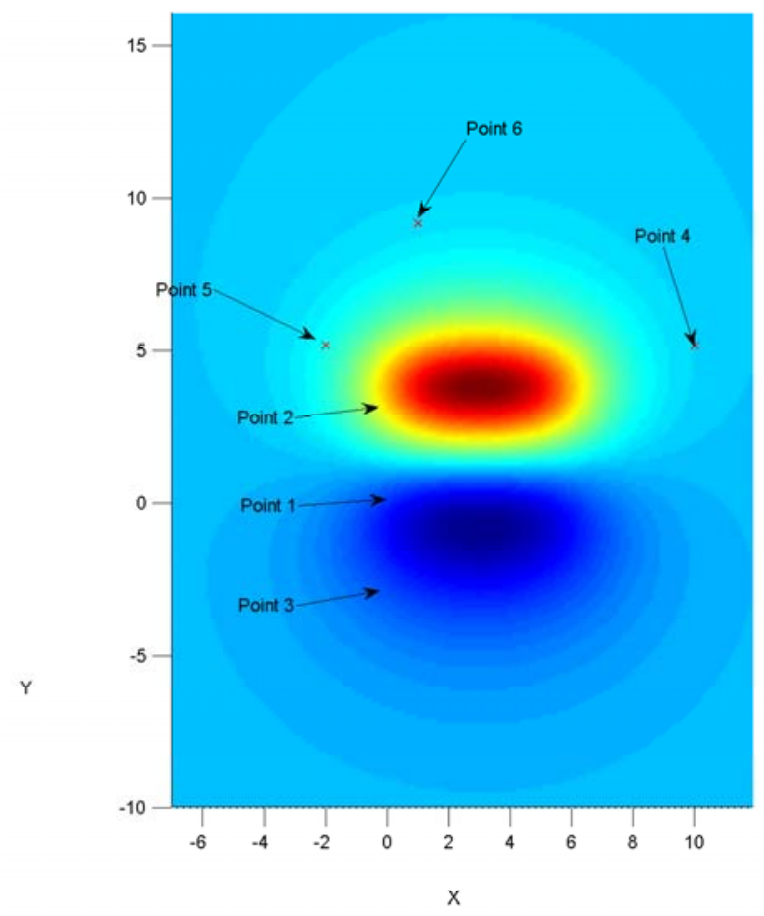

Figure 8: Top view of the initial free surface deformation showing the location of six selected gauges, with the following coordinates (in km): (1) 0,0 ; (2) 0,3 ; (3) $0,-3$; (4) 10,5 ; $(5)-2,5$; (6) 1,10 . The lower oval area represents the initial subsidence while the upper oval area represents the initial uplift. 

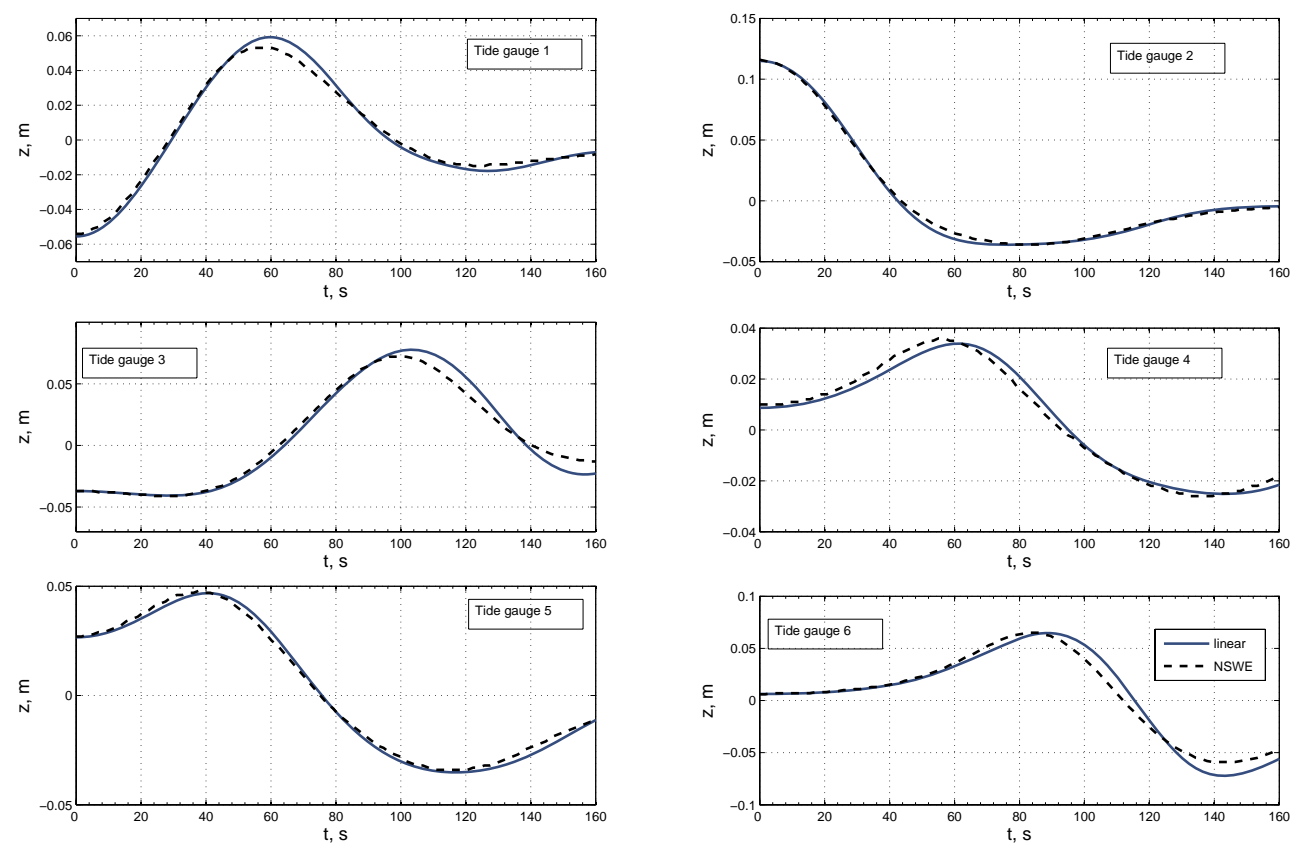

Figure 9: Transient waves generated by an underwater earthquake. Comparisons of the free-surface elevation as a function of time at the selected gauges shown in figure 8: - , linear model ; -- nonlinear shallow water model. The time $t$ is expressed in seconds. The physical parameters are those of figure 7 . Since the fully nonlinear results cannot be distinguished from the linear ones, they are not shown. 
with the three models for the same parameters as those of Figure 6, except for the water depth now equal to $1 \mathrm{~km}$. The small dimensionless numbers are roughly $\varepsilon=5 \times 10^{-5}$ and $\mu=0.1$, with a corresponding Ursell number equal to 0.005 . On one hand, linear and fully nonlinear models are essentially undistinguishable at all times: the waves propagate with the same speed and the same profile. Nonlinear effects are clearly negligible during the first moments of transient waves generated by a moving bottom, at least in this context. On the other hand, the numerical solution obtained with the NSW model gives slightly different results. Waves computed with this model do not propagate with the same speed and have different amplitudes compared to those obtained with the linear and fully nonlinear models. Dispersive effects come into the picture essentially because the waves are shorter compared to the water depth. As shown in the previous examples, dispersive effects do not play a role for long enough waves.

Figure 12 shows the transient waves at the gauges selected in figure 8 . One can see that the elevations obtained with the linear and fully nonlinear models are very close within graphical accuracy. On the contrary, the nonlinear shallow water model leads to a higher speed and the difference is obvious for the points away from the generation zone.

These results show that one cannot neglect the dispersive effects any longer. The NSW equations, which contain no dispersive effects, lead to different speed and amplitudes. Moreover, the oscillatory behaviour just behind the two front waves is no longer present. This oscillatory behaviour has been observed for the water waves computed with the linear and fully nonlinear models and is due to the presence of frequency dispersion. So, one should replace the NSW equations with Boussinesq models which combine the two fundamentals effects of nonlinearity and dispersion. Wei et al. [37] provided comparisons for twodimensional waves resulting from the integration of a Boussinesq model and the two-dimensional version of the FNPF model described above. In fact they used a fully nonlinear variant of the Boussinesq model, which predicts wave heights, phase speeds and particle kinematics more accurately than the standard weakly nonlinear approximation first derived by Peregrine [27] and improved by Nwogu's modified Boussinesq model [25]. We refer to the review [20] on Boussinesq models and their applications for a complete description of modern Boussinesq theory.

From a physical point of view, we emphasize that the wavelength of the tsunami waves is directly related to the mechanism of generation and to the dimensions of the source event. And so is the dimensionless number $\mu$ which determines the importance of the dispersive effects. In general it will remain small.

Adapting the discussion by Bona et al. [2], one can expect the solutions to the long wave models to be good approximations of the solutions to the full water-wave equations on a time scale of the order $\min \left(\varepsilon^{-1}, \mu^{-2}\right)$ and also the neglected effects to make an order-one relative contribution on a time scale of order $\min \left(\varepsilon^{-2}, \mu^{-4}, \varepsilon^{-1} \mu^{-2}\right)$. Even though we have not computed precisely the constant in front of these estimates, the results shown in this paper are in 

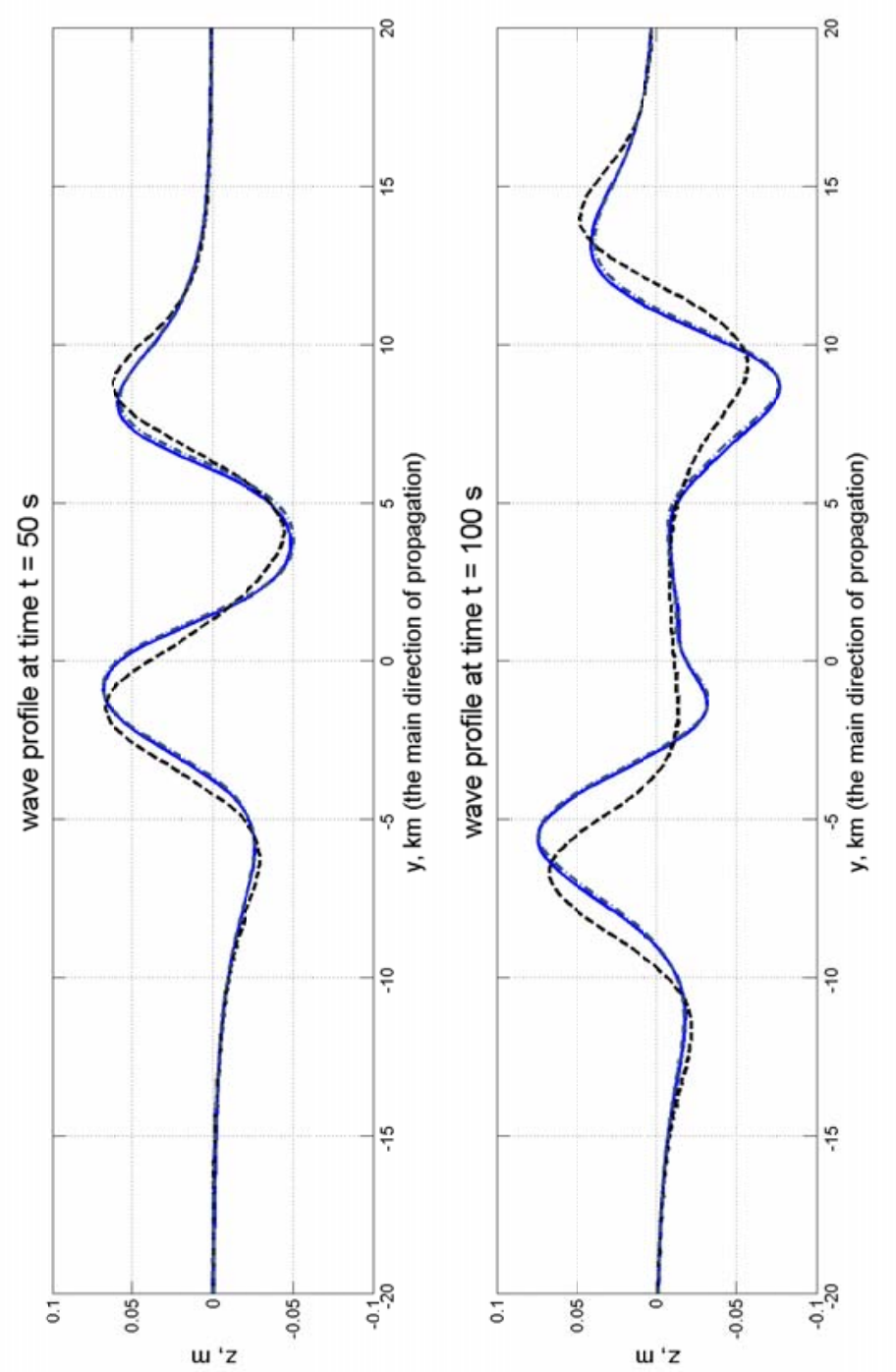

Figure 10: Comparisons of the free-surface elevation at $x=0$ resulting from the integration of the linear equations $(-\cdot-)$, NSW equations $(--)$ and FNPF equations $(-)$ at different times of the propagation of transient waves generated by an earthquake $(t=50 \mathrm{~s}, t=100 \mathrm{~s})$. The parameters for the earthquake are those given in Table 1 . The water depth is $1 \mathrm{~km}$. One has the following estimates: $\varepsilon=5 \times 10^{-5}, \mu^{2}=10^{-2}$ and consequently $S=0.005$. 

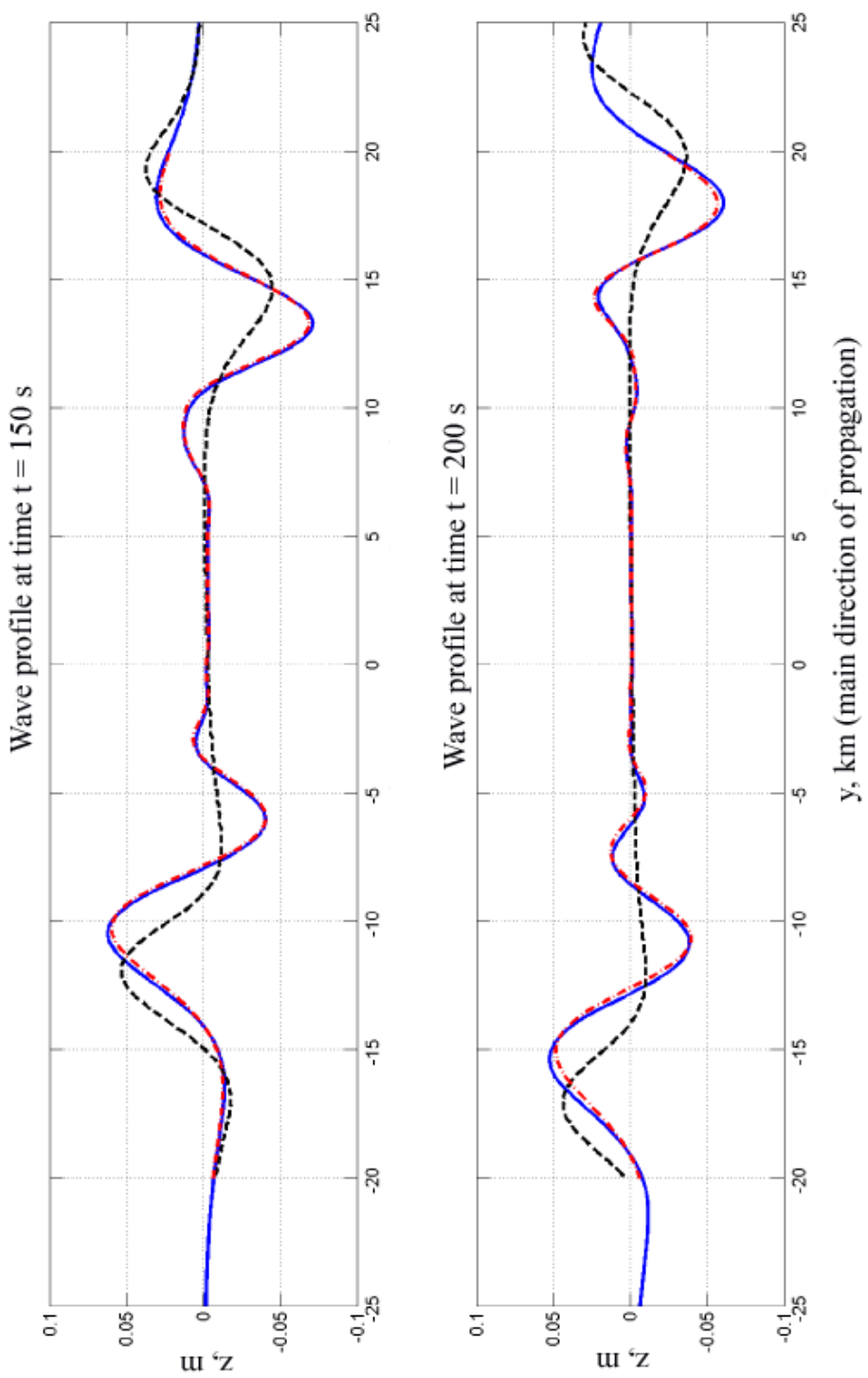

Figure 11: Same as figure 10 for later times $(t=150 \mathrm{~s}, t=200 \mathrm{~s})$. 

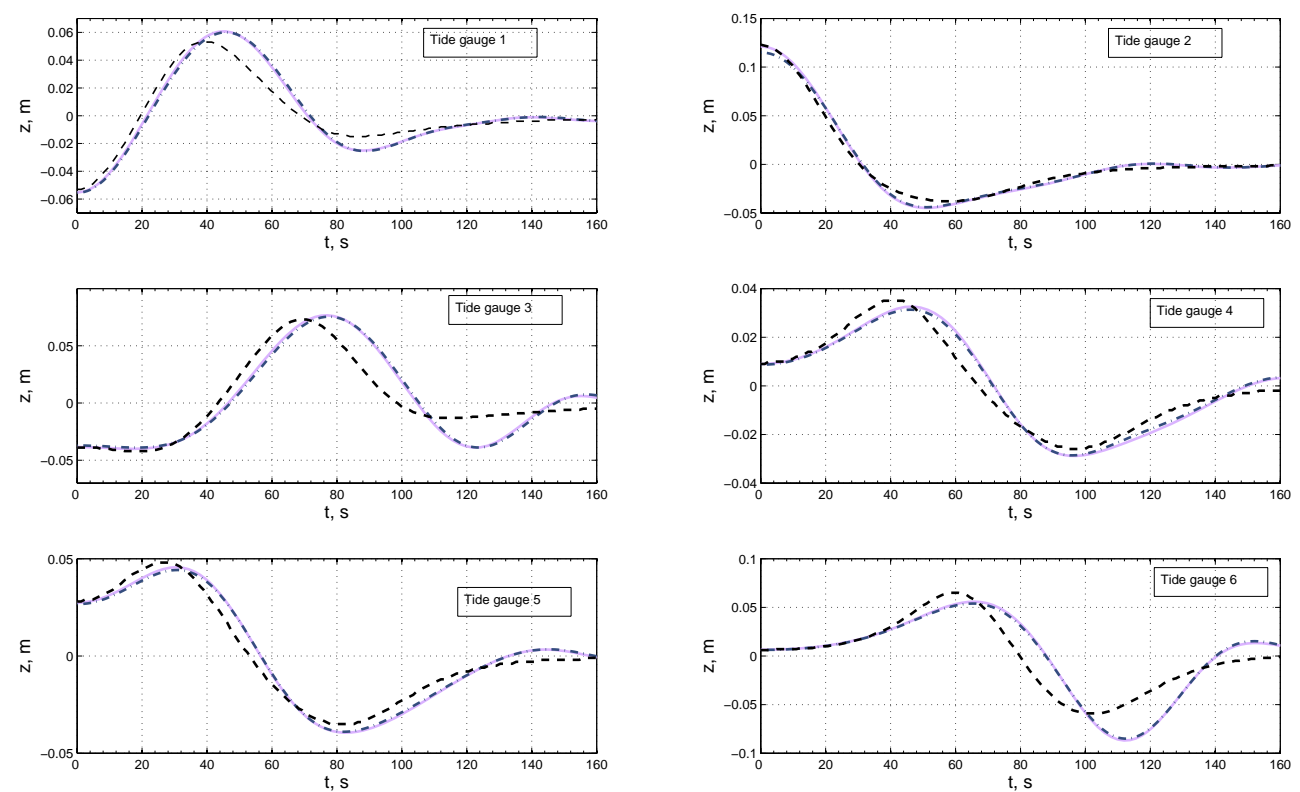

Figure 12: Transient waves generated by an underwater earthquake. The physical parameters are those of figures 10 and 11. Comparisons of the free-surface elevation as a function of time at the selected gauges shown in figure 8: -, linear model ; - nonlinear shallow water model. The time $t$ is expressed in seconds. The FNPF results cannot be distinguished from the linear results. 
agreement with these estimates. Considering the 2004 Boxing Day tsunami, it is clear that dispersive and nonlinear effects did not have sufficient time to develop during the first hours due to the extreme smallness of $\varepsilon$ and $\mu^{2}$, except of course when the tsunami waves approached the coast.

Let us conclude this section with a discussion on the generation methods, which extends the results given in $[5]^{6}$. We show the major differences between the classical passive approach and the active approach of wave generation by a moving bottom. Recall that the classical approach consists in translating the sea bed deformation to the free surface and letting it propagate. Results are presented for waves computed with the linear model.

Figure 13 shows the waves measured at several artificial gauges. The parameters are those of Table 1 , and the water depth is $h=500 \mathrm{~m}$. The solid line represents the solution with an instantaneous bottom deformation while the dashed line represents the passive wave generation scenario. Both scenarios give roughly the same wave profiles. Let us now consider a slightly different set of parameters: the only difference is the water depth which is now $h=1 \mathrm{~km}$. As shown in figure 14, the two generation models differ. The passive mechanism gives higher wave amplitudes.

Let us quantify this difference by considering the relative difference between the two mechanisms defined by

$$
r(x, y, t)=\frac{\left|\eta_{\text {active }}(x, y, t)-\eta_{\text {passive }}(x, y, t)\right|}{\left\|\eta_{\text {active }}\right\|_{\infty}} .
$$

Intuitively this quantity represents the deviation of the passive solution from the active one with a moving bottom in units of the maximum amplitude of $\eta_{\text {active }}(x, y, t)$.

Results are presented on figures (15) and (16). The differences can be easily explained by looking at the analytical formulas (25) and (39) of Section 3. These differences, which can be crucial for accurate tsunami modelling, are twofold.

First of all, the wave amplitudes obtained with the instantly moving bottom are lower than those generated by the passive approach (this statement follows from the inequality $\cosh m h \geq 1$ ). The numerical experiments show that this difference is about $6 \%$ in the first case and $20 \%$ in the second case.

The second feature is more subtle. The water column has an effect of a lowpass filter. In other words, if the initial deformation contains high frequencies, they will be attenuated in the moving bottom solution because of the presence of the hyperbolic $\operatorname{cosine} \cosh (m h)$ in the denominator which grows exponentially with $m$. Incidently, in the framework of the NSW equations, there is no difference between the passive and the active approach for an instantaneous seabed deformation $[32,33]$.

If we prescribe a more realistic bottom motion as in [4] for example, the results will depend on the characteristic time of the seabed deformation. When the characteristic time of the bottom motion decreases, the linearized solution

\footnotetext{
${ }^{6}$ In figures 1 and 2 of [5], a mistake was introduced in the time scale. All times must be multiplied by a factor $\sqrt{1000}$.
} 

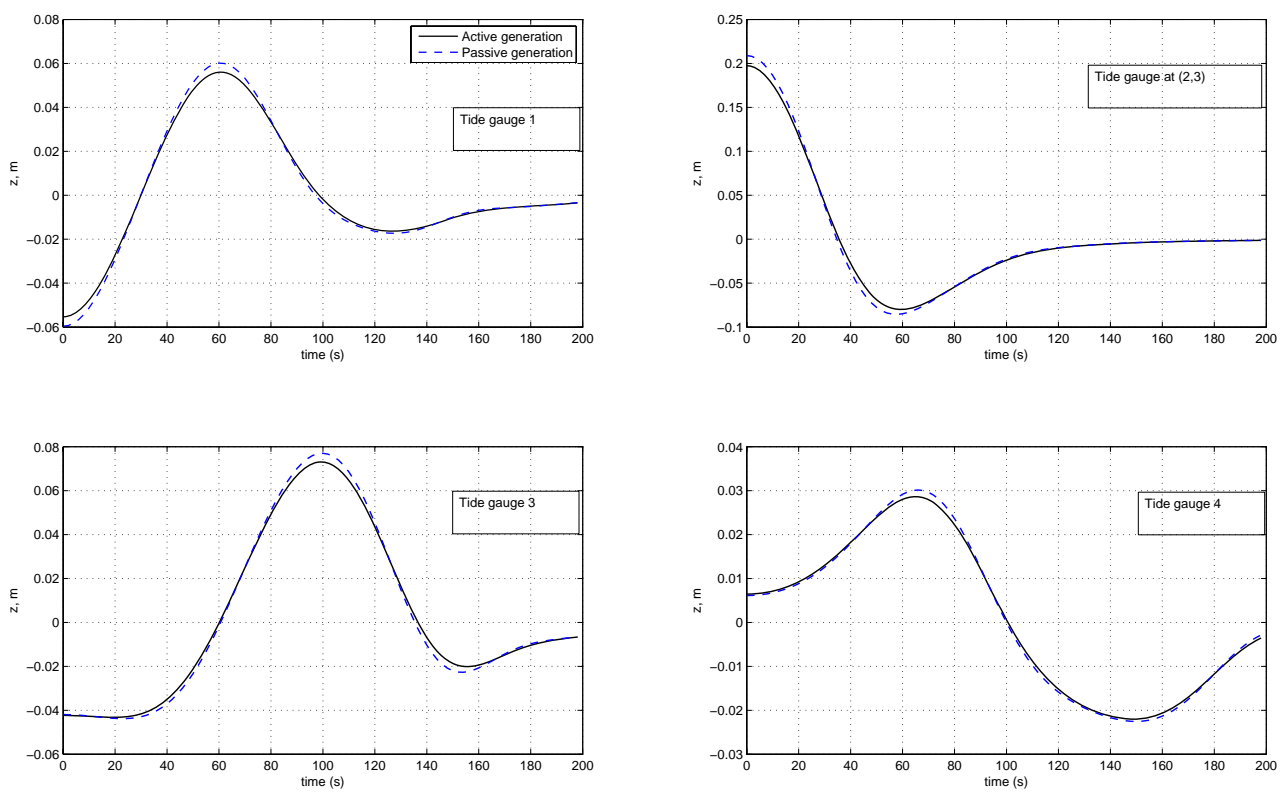

Figure 13: Transient waves generated by an underwater earthquake. The computations are based on linear wave theory. Comparisons of the free-surface elevation as a function of time at selected gauges for active and passive generation processes. The time $t$ is expressed in seconds. The physical parameters are those of figure 7. In particular, the water depth is $h=500 \mathrm{~m}$. 

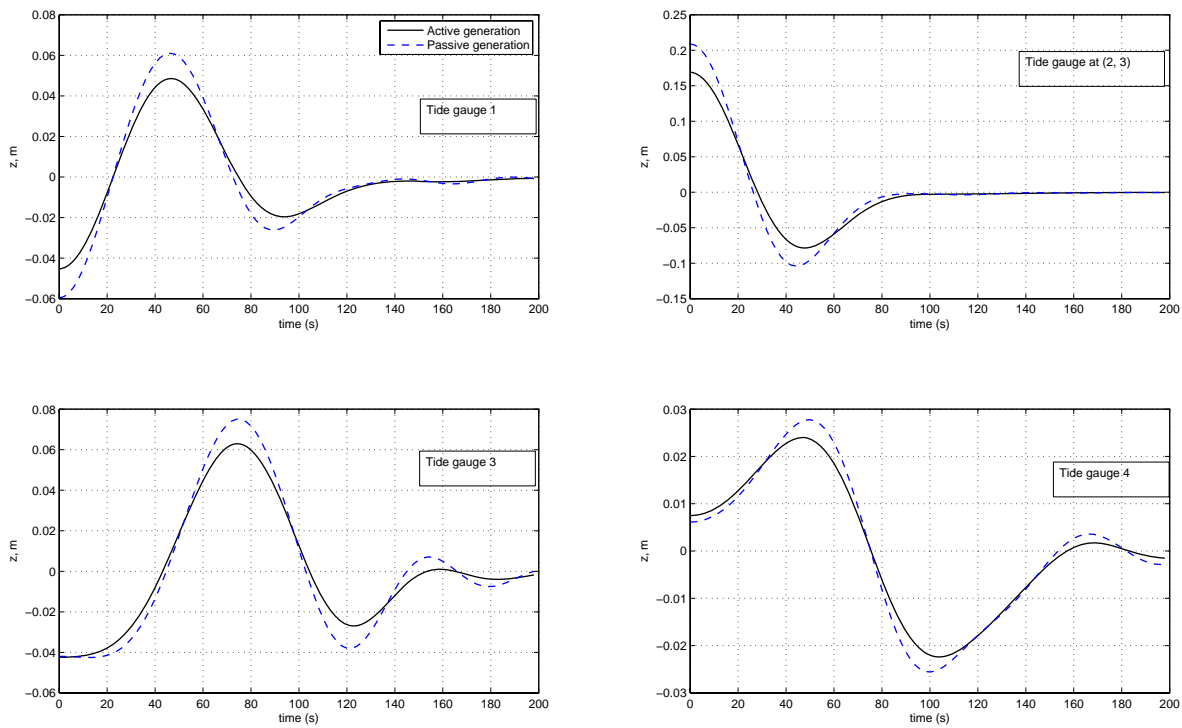

Figure 14: Same as figure 13, except for the water depth, which is equal to 1 $\mathrm{km}$.

tends to the instantaneous wave generation scenario. So, in the framework of linear water wave equations, one cannot exceed the passive generation amplitude with an active process. However, during slow events, Todorovska and Trifunac [31] have shown that amplification of one order of magnitude may occur when the sea floor uplift spreads with velocity similar to the long wave tsunami velocity.

\section{Conclusions}

Comparisons between linear and nonlinear models for tsunami generation by an underwater earthquake have been presented. There are two main conclusions that are of great importance for modelling the first instants of a tsunami and for providing an efficient initial condition to propagation models. To begin with, a very good agreement is observed from the superposition of plots of wave profiles computed with the linear and fully nonlinear models. Secondly, the nonlinear shallow water model was not sufficient to model some of the waves generated by a moving bottom because of the presence of frequency dispersion. However classical tsunami waves are much longer, compared to the water depth, than the waves considered in the present paper, so that the NSW model is also sufficient to describe tsunami generation by a moving bottom. Comparisons between the NSW equations and the FNPF equations for modeling tsunami run-up are left 

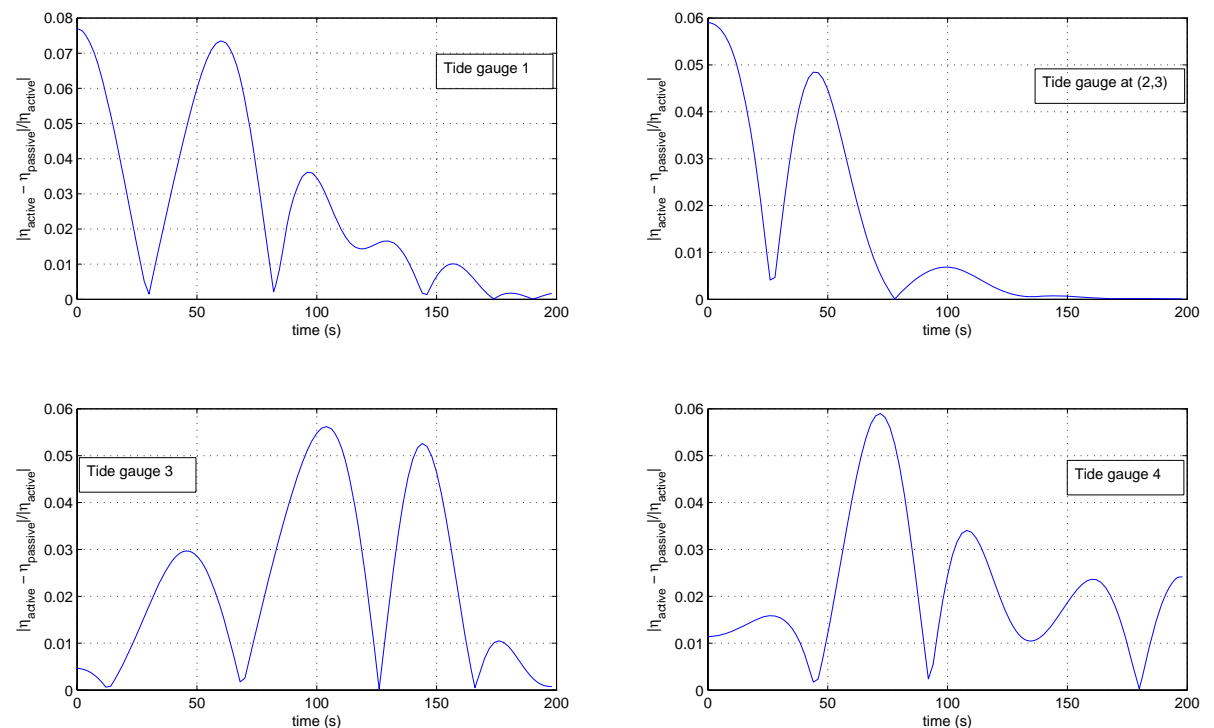

Figure 15: Relative difference between the two solutions shown in figure 13. The time $t$ is expressed in seconds.
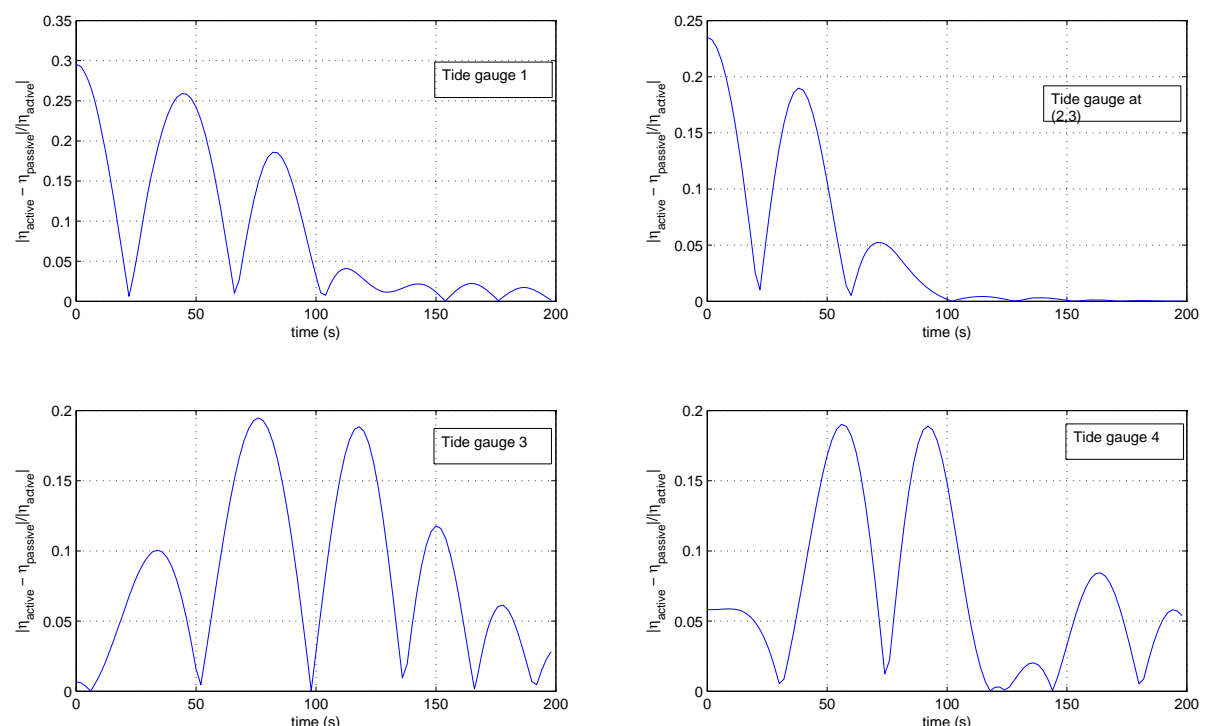

Figure 16: Relative difference between the two solutions shown in figure 14. 
for future work. Another aspect which deserves attention is the consideration of Earth rotation and the derivation of Boussinesq models in spherical coordinates.

\section{Acknowledgments}

The authors thank C. Fochesato for his help on the numerical method used to solve the fully nonlinear model. The first author gratefully acknowledges the kind assistance of the Centre de Mathématiques et de Leurs Applications of École Normale Supérieure de Cachan. The third author acknowledges the support from the EU project TRANSFER (Tsunami Risk ANd Strategies For the European Region) of the sixth Framework Programme under contract no. 037058 .

\section{References}

[1] Ben-Menahem A., Rosenman M. (1972) Amplitude patterns of tsunami waves from submarine earthquakes. J. Geophys. Res. 77:3097-3128

[2] Bona J.L., Colin T., Lannes D. (2005) Long wave approximations for water waves. Arch. Rational Mech. Anal. 178:373-410

[3] Bona J.L., Pritchard W.G., Scott L.R. (1981) An evaluation of a model equation for water waves. Phil. Trans. R. Soc. Lond. A 302:457-510

[4] Dutykh D., Dias F. (2007) Water waves generated by a moving bottom. In Tsunami and Nonlinear Waves, Ed: A. Kundu, Springer Verlag (Geo Sc.)

[5] Dutykh D., Dias F., Kervella Y. (2006) Linear theory of wave generation by a moving bottom. C. R. Acad. Sci. Paris, Ser. I, 343:499-504

[6] Filon L.N.G. (1928) On a quadrature formula for trigonometric integrals. Proc. Royal Soc. Edinburgh 49:38-47

[7] Fochesato C., Dias F. (2006) A fast method for nonlinear three-dimensional free-surface waves. Proc. R. Soc. A 462:2715-2735

[8] Geist E.L., Titov V.V., Synolakis C.E. (2006) Tsunami: wave of change. Scientific American 294:56-63

[9] Ghidaglia J.-M., Kumbaro A., Le Coq G. (1996) Une méthode volumes finis à flux caractéristiques pour la résolution numérique des systèmes hyperboliques de lois de conservation. C.R. Acad. Sc. Paris, Ser. I 322:981988

[10] Ghidaglia J.-M., Kumbaro A., Le Coq G. (2001) On the numerical solution to two fluid models via a cell centered finite volume method. Eur. J. Mech. B/Fluids 20:841-867 
[11] González F.I., Bernard E.N., Meinig C., Eble M.C., Mofjeld H.O., Stalin S. (2005) The NTHMP tsunameter network. Natural Hazards 35:25-39

[12] Grilli S., Guyenne P., Dias F. (2001) A fully non-linear model for threedimensional overturning waves over an arbitrary bottom. Int. J. Numer. Meth. Fluids 35:829-867

[13] Grilli S., Vogelmann S., Watts P. (2002) Development of a 3D numerical wave tank for modeling tsunami generation by underwater landslides. Engng Anal. Bound. Elem. 26:301-313

[14] Guesmia M., Heinrich P.H., Mariotti C. (1998) Numerical simulation of the 1969 Portuguese tsunami by a finite element method. Natural Hazards $17: 31-46$

[15] Hammack J.L. (1973) A note on tsunamis: their generation and propagation in an ocean of uniform depth. J. Fluid Mech. 60:769-799

[16] Hammack J.L., Segur H. (1974) The Korteweg-de Vries equation and water waves. Part 2. Comparison with experiments. J. Fluid Mech. 65:289-314

[17] Houston J.R., Garcia A.W. (1974) Type 16 flood insurance study. USACE WES Report No. H-74-3

[18] Kajiura K. (1963) The leading wave of tsunami. Bull. Earthquake Res. Inst., Tokyo Univ. 41:535-571

[19] Kânoglu K., Synolakis C. (2006) Initial value problem solution of nonlinear shallow water-wave equations. Phys. Rev. Lett. 97:148501

[20] Kirby J.T. (2003) Boussinesq models and applications to nearshore wave propagation, surfzone processes and wave-induced currents. In Advances in Coastal Modeling, V. C. Lakhan (ed), Elsevier, 1-41

[21] Kulikov E.A., Medvedev P.P., Lappo S.S. (2005) Satellite recording of the Indian Ocean tsunami on December 26, 2004. Doklady Earth Sciences A 401:444-448

[22] Lay T., Kanamori H., Ammon C.J., Nettles M., Ward S.N., Aster R.C., Beck S.L., Bilek S.L., Brudzinski M.R., Butler R., DeShon H.R., Ekstrom G., Satake K., Sipkin S. (2005) The great Sumatra-Andaman earthquake of 26 December 2004. Science 308:1127-1133

[23] LeVeque R.J. (1998) Balancing source terms and flux gradients in highresolution Godunov methods: the quasi-steady wave-propagation algorithm. J. Computational Phys. 146:346-365

[24] Liu P.L.-F., Liggett J.A. (1983) Applications of boundary element methods to problems of water waves, Chapter 3:37-67 
[25] Nwogu O. (1993) An alternative form of the Boussinesq equations for nearshore wave propagation. Coast. Ocean Engng. 119:618-638

[26] Okada Y. (1985) Surface deformation due to shear and tensile faults in a half-space. Bull. Seism. Soc. Am. 75:1135-1154

[27] Peregrine D.H. (1967) Long waves on a beach. J. Fluid Mech. 27:815-827

[28] Synolakis C.E., Bernard E.N. (2006) Tsunami science before and beyond Boxing Day 2004. Phil. Trans. R. Soc. A 364:2231-2265

[29] Titov V.V., Synolakis C.E. (1998) Numerical modeling of tidal wave runup. J. Waterway, Port, Coastal, and Ocean Engineering 124:157-171

[30] Todorovska M.I, Hayir A., Trifunac M.D. (2002) A note on tsunami amplitudes above submarine slides and slumps. Soil Dynamics and Earthquake Engineering 22:129-141

[31] Todorovska M.I., Trifunac M.D. (2001) Generation of tsunamis by a slowly spreading uplift of the sea-floor. Soil Dynamics and Earthquake Engineering 21:151-167

[32] Tuck E.O. (1979) Models for predicting tsunami propagation. NSF Workshop on Tsunamis, California, Ed: L.S. Hwang and Y.K. Lee, Tetra Tech Inc., 43-109

[33] Tuck E.O., Hwang L.-S. (1972) Long wave generation on a sloping beach. J. Fluid Mech. 51:449-461

[34] Ursell F. (1953) The long-wave paradox in the theory of gravity waves. Proc. Camb. Phil. Soc. 49:685-694

[35] Villeneuve M., Savage S.B. (1993) Nonlinear, dispersive, shallow-water waves developed by a moving bed. J. Hydraulic Res. 31:249-266

[36] Ward S.N. (2001) Landslide tsunami. J. Geophysical Res. 106:11201-11215

[37] Wei G., Kirby J.T., Grilli S.T., Subramanya R. (1995) A fully nonlinear Boussinesq model for surface waves. Part 1. Highly nonlinear unsteady waves. J. Fluid Mech. 294:71-92 